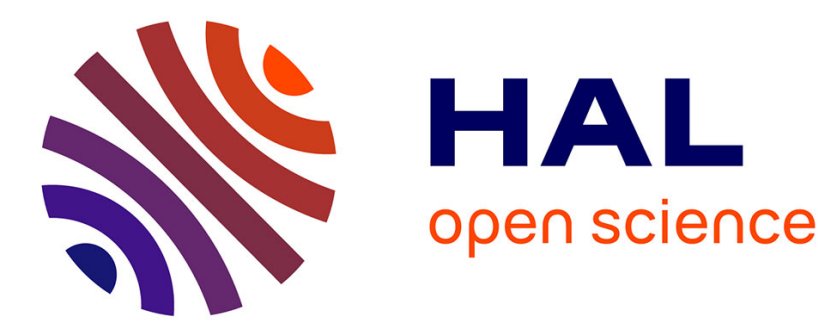

\title{
Du baccalauréat à l'enseignement supérieur : déplacement et recomposition des inégalités
}

Marie Duru-Bellat, Annick Kieffer

\section{To cite this version:}

Marie Duru-Bellat, Annick Kieffer. Du baccalauréat à l'enseignement supérieur: déplacement et recomposition des inégalités. Population (édition française), 2008, 63 (1), pp.123-157. hal-01022172

HAL Id: hal-01022172

https://hal-sciencespo.archives-ouvertes.fr/hal-01022172

Submitted on 10 Jul 2014

HAL is a multi-disciplinary open access archive for the deposit and dissemination of scientific research documents, whether they are published or not. The documents may come from teaching and research institutions in France or abroad, or from public or private research centers.
L'archive ouverte pluridisciplinaire HAL, est destinée au dépôt et à la diffusion de documents scientifiques de niveau recherche, publiés ou non, émanant des établissements d'enseignement et de recherche français ou étrangers, des laboratoires publics ou privés. 


\title{
DU BACCALAURÉAT À L'ENSEIGNEMENT SUPÉRIEUR EN FRANCE : DÉPLACEMENT ET RECOMPOSITION DES INÉGALITÉS
}

\author{
Marie Duru-Bellat et Annick Kieffer
}

I.N.E.D | Population

\author{
2008/1 - Vol. 63 \\ pages 123 à 157
}

ISSN 0032-4663

Article disponible en ligne à l'adresse:

http://www.cairn.info/revue-population-2008-1-page-123.htm

Pour citer cet article :

Duru-Bellat Marie et Kieffer Annick, « Du baccalauréat à l'enseignement supérieur en France : déplacement et recomposition des inégalités ",

Population, 2008/1 Vol. 63, p. 123-157. DOI : 10.3917/popu.801.0123

Distribution électronique Cairn.info pour I.N.E.D.

(C) I.N.E.D. Tous droits réservés pour tous pays.

La reproduction ou représentation de cet article, notamment par photocopie, n'est autorisée que dans les limites des conditions générales d'utilisation du site ou, le cas échéant, des conditions générales de la licence souscrite par votre établissement. Toute autre reproduction ou représentation, en tout ou partie, sous quelque forme et de quelque manière que ce soit, est interdite sauf accord préalable et écrit de l'éditeur, en dehors des cas prévus par la législation en vigueur en France. II est précisé que son stockage dans une base de données est également interdit. 


\title{
MARIE DURU-BelLAT* ET ANNICK KIEFFER**
}

\section{Du baccalauréat à l'enseignement supérieur en France : déplacement et recomposition des inégalités}

\begin{abstract}
La question de la démocratisation de l'enseignement en France est un sujet de débats : si les taux de scolarisation n'ont pas cessé de croître, ce constat quantitatif global se traduit-il véritablement par une réduction des inégalités sociales dans l'accès aux différents niveaux et aux différentes filières scolaires et universitaires? Faisant suite à un article publié dans Population en 2000 et dont le titre évoquait cet objet de polémique, MARIE DURU-BELLAT et ANNICK KIEFFER proposent ici une analyse précise des mécanismes de reproduction des inégalités au sein de l'enseignement supérieur en France. Ces résultats apportent indéniablement des éléments critiques à la discussion en montrant que l'ouverture quantitative du système éducatif s'accompagne toujours d'une différenciation sociale marquée des étudiants selon les filières et les disciplines.
\end{abstract}

Le thème de la démocratisation de l'enseignement est particulièrement nourri dans la recherche française en sciences sociales, ce qui ne rend pas moins nécessaire une réactualisation continue des données et des interprétations au fil du temps, selon les évolutions qui affectent les différents niveaux d'enseignement. À cet égard, la période 1985-1995 est particulièrement intéressante, puisqu'elle a été marquée par une augmentation spectaculaire de la proportion d'une classe d'âge obtenant le baccalauréat, c'est-à-dire parvenant diplômée au terme de l'enseignement secondaire long (entre 17 et 19 ans). Rappelons en effet que la proportion de bacheliers dans une génération, qui était de l'ordre de $5 \%$ en 1950, est passée de $20 \%$ en 1970 à 29,4\% en 1985 , puis à 43,5\% en 1990 et à 62,7\% en 1995 . Elle stagne depuis cette date.

Ce changement d'échelle considérable du nombre de jeunes terminant avec succès des études secondaires s'est produit au sein d'un système qui s'est diversifié. Dès lors qu'en France le baccalauréat est un titre qui ouvre de droit les

\footnotetext{
* Université de Bourgogne-CNRS, Iredu.

** CNRS, Centre Maurice Halbwachs.
} 
portes de l'enseignement supérieur, les effectifs d'étudiants ont crû également de façon très forte pendant la période, conduisant à un doublement du nombre de diplômés entre 1985 et 1996. Dans ce contexte de fort changement qui semble aujourd'hui stabilisé, il est intéressant d'évaluer les formes qu'a revêtues la démocratisation de l'enseignement au niveau du supérieur, et donc d'examiner avec précision les modifications des cursus des étudiants en fonction de leurs caractéristiques personnelles.

Dans tous les pays européens, l'enseignement supérieur s'est fortement développé, dans un contexte où les instances européennes mettaient en avant la nécessité de favoriser l'essor d'une « société de la connaissance ». Ce développement « quantitatif » s'est le plus souvent accompagné d'une diversification des structures. Censée permettre un appariement plus efficace entre les « offres » pédagogiques et les publics plus variés qu'il s'agit à présent de scolariser, cette diversification autorise aussi une hiérarchisation croissante des formations qui constitue un enjeu social. La problématique dominante dans la sociologie européenne consiste dès lors à se demander si les inégalités sociales face à l'enseignement supérieur (et donc d'accès à un statut social supérieur) ne sont pas en train de se modifier profondément : elles se joueraient davantage dans l'accès à telle ou telle filière que dans l'accès ou non à ce niveau d'enseignement ; la différenciation sociale des filières prendrait alors le pas sur la différenciation des niveaux. Certains vont même jusqu'à avancer que «l'élargissement de l'accès à l'enseignement prend la forme d'une différenciation hiérarchisée, qui conduit les membres des classes populaires vers des filières moins valorisées et réserve les filières les plus prestigieuses aux groupes sociaux supérieurs » (Arum et al., 2007, p. 5).

On dispose à ce sujet d'analyses récentes pour la France (Merle, 1996, 2000 et 2002 ; Albouy et Wanecq, 2003 ; Selz et Vallet, 2006), mais les transformations tout à fait considérables qu'ont connues les diverses filières de l'enseignement secondaire et celles de l'enseignement supérieur pendant les deux dernières décennies restent encore insuffisamment explorées, notamment en ce qui concerne l'évolution des choix d'études effectués à ce dernier niveau. Seule leur prise en compte peut permettre d'évaluer, pour ce qui est de l'enseignement supérieur qui devient le niveau de sortie du système éducatif d'un jeune sur deux, dans quelle mesure et selon quelles modalités une démocratisation est advenue, en distinguant, selon la typologie de Merle (2000), une démocratisation égalisatrice (permettant aux écarts entre groupes de se resserrer), une démocratisation uniforme (où les écarts sont simplement déplacés à des niveaux de scolarisation plus élevés) et une démocratisation ségrégative, où les inégalités s'immiscent dans la différenciation des types d'études et des spécialités. Une question corollaire est d'examiner comment s'articulent aujourd'hui les inégalités qui résultent de la longueur des études et celles qui passent par leur type et leur spécialité. 
On ne saurait traiter cette question de l'évolution de la sélectivité sociale dans l'enseignement supérieur sans prendre en compte celle de la population des « éligibles », et donc intégrer dans l'analyse les transformations quantitatives et qualitatives du public bachelier. Nous le ferons dans une première partie, avant d'examiner successivement le passage entre les niveaux secondaire et supérieur, puis les orientations à ce niveau, en distinguant les types d'études et leur spécialité. Nous nous appuierons sur la dernière enquête Formation et Qualification Professionnelle (FQP) conduite par l'Insee en $2003^{(1)}$.

\section{L'évolution de l'accès au baccalauréat}

\section{Un contexte général de démocratisation}

Rappelons qu'en France, dans un contexte historique de développement de la scolarisation, on a observé une tendance très nette à la démocratisation au niveau de l'accès à l'enseignement secondaire, qui s'est accompagnée d'un déplacement des inégalités, de l'entrée en $6^{\mathrm{e}}$ à l'entrée en $2^{\text {nde }}$ au cours des décennies 1970 et $1980^{(2)}$ (Duru-Bellat et Kieffer, 2000 ; voir aussi Vallet, 2001). C'est seulement dans la période récente que l'accès au second cycle de l'enseignement secondaire s'est ouvert : on n'observe aucune baisse des inégalités d'accès au lycée entre les enfants de cadres et les enfants d'ouvriers entrés en $6^{e}$ en 1972 et en $1980^{(3)}$, alors qu'une baisse significative intervient quand on compare les scolarités des élèves entrés en $6^{\mathrm{e}}$ en 1980 et en 1989. Au seuil de cette période, les taux d'accès au lycée étaient d'environ $90 \%$ pour les enfants de cadres et de $42 \%$ pour les enfants d'ouvriers, et il était donc logique que toute nouvelle expansion se traduise par une poursuite du resserrement des écarts par le jeu d'« effets de plafond » concernant les catégories les plus favorisées. C'est ce que Merle (2000) appelle une " démocratisation égalisatrice».

Le développement des scolarités dans le secondaire découle d'une politique explicite, proclamée au début des années 1980 : « 80 \% d’une génération au niveau du baccalauréat ». Elle s'est traduite par une baisse des redoublements dès l'enseignement primaire et une suppression des orientations précoces vers

(1) C'est la sixième enquête d'une série qui a débuté en 1964. Cette enquête est réalisée par l'Institut national de la statistique et des études économiques (Insee) auprès des individus âgés de 18 à 65 ans vivant en ménage, résidant en France. Elle comprend un volet biographique qui fournit une description détaillée du cursus scolaire, année après année, ainsi que des indications sur la catégorie socioprofessionnelle et le niveau d'instruction de chacun des parents de la personne enquêtée. Enfin, elle fournit des indications détaillées sur la situation professionnelle des individus, à l'entrée dans la vie active, au moment de l'enquête et 5 ans auparavant. La cohorte 1962-1967, âgée de 36 à 41 ans en 2003, comprend 5984 personnes; la cohorte 1975-1980, âgée de 23 à 28 ans à la même date, en comprend 3889 .

(2) En France, l'enseignement secondaire est dispensé dans les collèges (premier cycle : de la classe

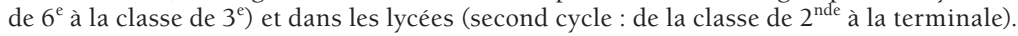

(3) Données issues des panels d'élèves 1972, 1980, 1989 et 1995, suivis par le ministère de l'Éducation nationale à partir de leur entrée dans le premier cycle du secondaire, enseignement indifférencié qui a accueilli progressivement tous les élèves au cours des années 1960. 
l'enseignement professionnel qui, jusqu'au milieu des années 1980, conduisaient les élèves les plus faibles mais aussi plus souvent les élèves des milieux populaires à quitter, dès 14-15 ans, l'enseignement secondaire. On peut donc conclure à une baisse de la sélectivité des carrières scolaires, sans que dans le même temps le niveau académique des élèves ait connu une amélioration significative ; cela a amené certains auteurs à parler d'une relative sous-sélection des élèves de milieu populaire susceptible d'affecter leurs cursus ultérieurs (Convert, 2006). Toujours est-il qu'accède à l'enseignement secondaire long une population plus hétérogène, amenée à s'orienter dans un système qui s'est dans le même temps fortement diversifié. En particulier, un nouveau baccalauréat, le baccalauréat professionnel, a été créé en 1985 et délivré pour la première fois en 1987-1988 pour conduire en deux ans à ce diplôme les jeunes qui après la troisième avaient été orientés vers la filière professionnelle de l'enseignement secondaire $^{(4)}$. Il était évident qu'on ne pouvait atteindre l'objectif d'amener $80 \%$ d'une génération au niveau du baccalauréat sans puiser dans ce vivier de jeunes au profil académique plus faible et d'origine plus populaire ${ }^{(5)}$.

Comme le montre le tableau 1, le taux d'accès au baccalauréat général a cessé de croître depuis 1995 alors qu'il avait plus que doublé depuis 1970. Si la hausse a été forte pour tous les types de baccalauréat entre 1985 et 1995, on observe que le taux d'accès au baccalauréat général a connu un certain tassement depuis, tandis que ce taux a continué à croître légèrement avant de se stabiliser pour les baccalauréats technologiques et professionnels. Ces baccalauréats, technologiques et plus encore professionnels, vont de fait s'adresser aux élèves les moins bons sur le plan scolaire : compte tenu du fonctionnement global de l'orientation en France, tous les élèves dont le niveau le permet choisissent de rester le plus longtemps possible dans les filières générales. Dès lors que les élèves de milieu populaire sont plus nombreux à rencontrer des difficultés scolaires, ils vont être surreprésentés dans ces filières technologiques et professionnelles : les enfants d'ouvriers constituent ainsi $14 \%$ des effectifs des filières générales, $26 \%$ de ceux des filières technologiques et $36 \%$ de ceux des filières professionnelles. Les enfants de chômeurs et d'inactifs représentent respectivement $5 \%, 8 \%$ et $12 \%$ des effectifs de ces filières.

(4) À la sortie du collège (classe de $3^{\mathrm{e}}$ ) les élèves sont orientés dans une classe de seconde indifférenciée ou préparent un diplôme professionnel en deux ans, le brevet d'études professionnelles (BEP). À la fin de la seconde, les élèves préparent en deux années soit un baccalauréat général qui sanctionne une formation à dominante scientifique, ou économique et sociale, ou littéraire, soit un baccalauréat technologique qui associe une formation générale à une formation couvrant un champ technologique d'ensemble. Les élèves qui ont suivi une filière professionnelle peuvent à l'issue du BEP préparer un baccalauréat professionnel en deux ans. Cette filière délivre une formation concrète débouchant sur un métier, même si, comme tout baccalauréat, il donne le droit de poursuivre dans l'enseignement supérieur.

(5) Cette réforme a également été justifiée par une volonté politique d'augmenter le niveau de qualification des ouvriers et des employés pour faire face aux évolutions technologiques. 
Tableau 1. Proportion d'une génération obtenant le baccalauréat selon le type de baccalauréat (\%)

\begin{tabular}{|l|c|c|c|c|}
\hline & $\begin{array}{c}\text { Baccalauréat } \\
\text { général }\end{array}$ & $\begin{array}{c}\text { Baccalauréat } \\
\text { technologique }\end{array}$ & $\begin{array}{c}\text { Baccalauréat } \\
\text { professionnel }\end{array}$ & Ensemble \\
\hline 1970 & 16,7 & 3,4 & & 20,1 \\
1985 & 19,8 & 9,6 & 2,8 & 29,4 \\
1990 & 27,9 & 12,8 & 7,9 & 43,5 \\
1995 & 37,2 & 17,6 & 11,4 & 62,7 \\
2000 & 32,9 & 18,5 & 11,5 & 62,8 \\
2005 & 33,7 & 17,3 & 62,5 \\
\hline Note : Les pourcentages sont obtenus en rapportant, pour chaque âge, le nombre de titulaires du baccalauréat \\
à la population totale de cet âge, pour les années considérées. \\
Source : Ministère de l'Éducation nationale (Repères et Références statistiques, éd. 2006). \\
\hline
\end{tabular}

Il est donc fondé, comme le propose Merle (2000 et 2002), de parler de " démocratisation ségrégative », dès lors que l'ouverture de l'accès au baccalauréat s'est réalisée par le développement différencié de l'accès à ses diverses séries ; ces différences peuvent être considérées comme des inégalités, dans la perspective qui est la nôtre ici, en ce sens que les taux de passage dans l'enseignement supérieur et les taux de réussite à ce niveau s'avèrent fortement corrélés avec le type de baccalauréat obtenu, depuis les séries générales, de loin les mieux placées à cet égard, jusqu'aux séries professionnelles.

\section{L'évolution des inégalités d'accès au baccalauréat dans la période récente}

Pour évaluer plus précisément ces évolutions, nous avons isolé deux groupes de générations dans l'enquête FQP, soit les générations 1962-1967 (qui ont obtenu, le cas échéant, le baccalauréat au début des années 1980) et les générations 1975-1980 (qui l'ont obtenu autour de 1995); ces deux cohortes ont été retenues pour « cadrer » la période où la proportion de bacheliers et le taux d'accès à l'enseignement supérieur ont le plus augmenté ${ }^{(6)}$. Nous distinguerons la probabilité d'obtenir un baccalauréat (quel qu'il soit), d'une part, et la probabilité d'obtenir tel ou tel type de baccalauréat d'autre part (général, technologique ou professionnel).

Pour mesurer l'ampleur des inégalités, nous utiliserons les deux types d'indicateurs courants dans la littérature, d'une part des différences entre taux (méthode additive), d'autre part des rapports des chances (odds ratios). Sans reprendre ici les débats autour de l'opportunité et du sens de ces deux types

(6) En revanche, quand il s'agira d'analyser non plus l'accès à l'enseignement supérieur mais l'obtention " finale » d'un diplôme, nous avons utilisé des générations légèrement plus anciennes (1972-1977 au lieu des générations 1975-1980) pour tenir compte des poursuites d'études à un âge élevé (27\% des jeunes entrés dans l'enseignement supérieur dans la cohorte 1975-1980 sont encore étudiants en 2003, contre seulement 5 \% dans la cohorte 1972-1977). 
de mesure (pour une discussion approfondie, cf. Duru-Bellat et Kieffer, 2000 ; Manzo, 2006), contentons-nous de rappeler que les premiers sont pertinents dans une perspective selon laquelle toute ouverture qui élargit l'accès à un bien éducatif doit être considérée comme une démocratisation, parce que l'éducation est un bien en soi ; il est alors justifié de s'intéresser à la quantité d'éducation reçue par chacun. Les seconds s'inscrivent dans une perspective différente, selon laquelle l'éducation est un bien relatif - qui ne vaut que par rapport à ce qu'ont les autres; ce sont donc les vitesses relatives d'évolution des taux d'accès des uns et des autres qui importent et, en filigrane, l'évolution de la compétition entre groupes. Au niveau scolaire qui nous intéresse, le baccalauréat, les deux perspectives ont chacune leur légitimité( ${ }^{(7)}$ : le baccalauréat a en effet, en soi, une forte valeur symbolique en France et sanctionne des savoirs dont la valeur est intrinsèque ; mais, dès lors que ce sont ses débouchés qui sont pris en compte, une approche relative est plus appropriée.

Le tableau 2 présente l'évolution des taux d'accès aux différents types de baccalauréat entre les deux cohortes en opposant les groupes les plus extrêmes, les enfants de cadres (qui représentent $6,4 \%$ des effectifs dans la première cohorte et $14,6 \%$ dans la seconde) et les enfants d'ouvriers (respectivement $39,3$ et $36,4 \%)$.

\section{Tableau 2. Évolution de l'accès des enfants d'ouvriers aux différents types de baccalauréat comparé à celui des enfants de cadres}

\begin{tabular}{|c|c|c|c|c|c|}
\hline & \multicolumn{3}{|c|}{$\begin{array}{l}\text { Proportion de bacheliers } \\
\text { (en \%) }\end{array}$} & \multicolumn{2}{|c|}{$\begin{array}{l}\text { Écart entre enfants } \\
\text { de cadres et enfants } \\
\text { d'ouvriers }\end{array}$} \\
\hline & Tous & $\begin{array}{l}\text { Enfants } \\
\text { de cadres }\end{array}$ & $\begin{array}{c}\text { Enfants } \\
\text { d'ouvriers }\end{array}$ & $\begin{array}{c}\text { Différences de } \\
\text { pourcentage } \\
\text { (en points) }\end{array}$ & Odds ratios \\
\hline \multicolumn{6}{|l|}{ Cohorte 1962-1967 } \\
\hline Ensemble des bacheliers & 35,0 & 74,5 & 20,6 & 53,9 & 11,3 \\
\hline Bacheliers généraux & 22,0 & 60,9 & 9,8 & 51,1 & 14,3 \\
\hline $\begin{array}{l}\text { Bacheliers technologiques } \\
\text { ou professionnels }\end{array}$ & 13,0 & 13,6 & 10,8 & 2,8 & 1,3 \\
\hline \multicolumn{6}{|l|}{ Cohorte 1975-1980 } \\
\hline Ensemble des bacheliers & 63,4 & 89,2 & 56,6 & 32,6 & 6,3 \\
\hline Bacheliers généraux & 38,6 & 74,0 & 20,2 & 53,8 & 11,2 \\
\hline $\begin{array}{l}\text { Bacheliers technologiques } \\
\text { ou professionnels }\end{array}$ & 24,9 & 15,2 & 36,4 & $-21,2$ & 0,3 \\
\hline
\end{tabular}

(7) Rappelons que les différences de pourcentage sont très sensibles au niveau des chiffres initiaux (dont les odds ratios sont quant à eux indépendants). 
Avoir ou non un bac...

Les odds ratios confirment tout d'abord une certaine démocratisation de l'accès au baccalauréat, quand on ne tient pas compte du type de baccalauréat obtenu : ils baissent de 11,3 à 6,3 (en opposant enfants de cadres et enfants d'ouvriers). Cette évolution est visible également (elle apparaît même beaucoup plus spectaculaire) sur la base des différences de pourcentage puisque l'écart entre les deux groupes passe de 54 à 33 points : même si la compétition ne s'atténue que légèrement, la croissance des taux rend l'obtention de ce « bien » (le baccalauréat) moins inégalitaire.

Des régressions logistiques permettant de raisonner « toutes choses égales par ailleurs » confirment ce premier constat (tableau 3). Entre les deux cohortes, les coefficients exprimant l'impact de la profession paternelle sur les chances d'obtenir un baccalauréat sont en baisse (tout en restant significatifs en fin de période) ; on observe en particulier une baisse de l'avantage des enfants de cadres et, de manière moins marquée, de celui des enfants de professions intermédiaires et d'agriculteurs ; on note également, toujours par rapport aux enfants d'ouvriers pris comme référence, que les enfants d'employés se rapprochent sensiblement, dans tous les modèles, de cette catégorie. En ce qui concerne l'influence du niveau d'instruction des parents, le fait d'avoir un père doté d'un diplôme du supérieur apporte en revanche un avantage important et sensiblement croissant. Par ailleurs, l'écart entre les enfants dont les pères n'ont pas de diplôme et ceux qui sont dotés d'un CAP ou d'un BEP se resserre légèrement, ce qui est sans doute lié à la baisse de la place relative des CAP dans la hiérarchie des niveaux d'instruction dans la génération des parents. Rappelons que le poids des pères non diplômés a baissé sur la période (de $60 \%$ à $42 \%$ ), ce qui accentue le caractère typé de ce groupe.

L'influence de la profession de la mère semble plus importante dans la seconde cohorte (notamment pour ce qui est de l'avantage apporté par une mère cadre), non sans lien, sans doute, avec le développement de l'activité féminine. Mais l'impact du niveau d'instruction de la mère, quant à lui, n'augmente pas (à moins qu'il ne soit de plus en plus " absorbé " par l'effet de la profession). Quoi qu'il en soit, ces résultats rejoignent certains constats antérieurs, selon lesquels la structure des inégalités sociales pourrait s'être modifiée ces dernières années, avec une influence décroissante de la profession du père et une influence croissante de son niveau d'instruction (voir par exemple Goux et Maurin, 1997). Les inégalités changeraient donc de forme plus qu'elles ne disparaîtraient, ainsi sans doute que les mécanismes qui les engendrent.

Il convient de souligner le caractère récent de cette évolution. Des modèles comparables estimés pour des générations nées à partir des années 1930 montraient une stabilité globale des inégalités d'accès au baccalauréat jusqu'aux cohortes nées à la fin des années 1950 (Duru-Bellat et Kieffer, 2000). Par contraste, entre les deux cohortes prises en compte ici, on assiste à des changements importants, qui accompagnent l'accroissement spectaculaire du taux d'accès au bac. 
Tableau 3. Évolution de l'accès au baccalauréat et à un baccalauréat général sur l'ensemble de la cohorte et de l'accès à un baccalauréat général dans la population bachelière (coefficients des modèles logit)

\begin{tabular}{|c|c|c|c|c|c|c|}
\hline & \multicolumn{2}{|c|}{$\begin{array}{l}\text { Modèle } 1 \\
\text { Tout baccalauréat } \\
\text { (ensemble de la } \\
\text { population) }\end{array}$} & \multicolumn{2}{|c|}{$\begin{array}{l}\text { Modèle } 2 \\
\text { Baccalauréat } \\
\text { général (ensemble } \\
\text { de la population) }\end{array}$} & \multicolumn{2}{|c|}{$\begin{array}{l}\text { Modèle } 3 \\
\text { Baccalauréat } \\
\text { général parmi les } \\
\text { bacheliers }\end{array}$} \\
\hline & $\begin{array}{c}\text { Cohorte } \\
1962-1967\end{array}$ & \begin{tabular}{|c|} 
Cohorte \\
$1975-1980$
\end{tabular} & $\begin{array}{l}\text { Cohorte } \\
1962-1967\end{array}$ & $\begin{array}{l}\text { Cohorte } \\
1975-1980\end{array}$ & $\begin{array}{l}\text { Cohorte } \\
1962-1967\end{array}$ & $\begin{array}{l}\text { Cohorte } \\
1975-1980\end{array}$ \\
\hline \multicolumn{7}{|l|}{ Profession du père } \\
\hline Ouvrier (réf.) & 0 & 0 & 0 & 0 & 0 & 0 \\
\hline Agriculteur & $0,56 * * *$ & $-0,10$ & 0,35 & 0,32 & $-0,14$ & 0,42 \\
\hline $\begin{array}{l}\text { Artisan, commerçant, } \\
\text { chef d'entreprise }\end{array}$ & $0,72 * * *$ & $0,67 * * *$ & $0,74 * * *$ & $0,95^{* * *}$ & 0,24 & $0,82 * * *$ \\
\hline Cadre & $1,44^{* * *}$ & $0,82 * * *$ & $1,46^{* * *}$ & $1,10 * * *$ & $0,71 * * *$ & $0,94 * * *$ \\
\hline $\begin{array}{l}\text { Profession } \\
\text { intermédiaire }\end{array}$ & $0,96 * * *$ & $0,75^{\star * *}$ & $1,03 * * *$ & $0,68 * * *$ & $0,49 * * *$ & $0,44^{* \star}$ \\
\hline Employé & $0,45^{* * *}$ & $0,22 *$ & $0,71 * * *$ & $0,34 * * *$ & $0,63 * * *$ & $0,28^{*}$ \\
\hline Autre & 0,11 & $-0,35^{\star}$ & 0,54 ** & $-0,64 * * *$ & 0,90 ** & $-0,62^{*}$ \\
\hline \multicolumn{7}{|l|}{ Profession de la mère } \\
\hline Inactive (réf.) & 0 & 0 & 0 & 0 & 0 & 0 \\
\hline Agricultrice & 0,10 & $1,10 * *$ & 0,09 & 0,32 & 0,03 & $-0,29$ \\
\hline $\begin{array}{l}\text { Artisane, commerçante, } \\
\text { chef d'entreprise }\end{array}$ & 0,10 & 0,09 & 0,08 & $-0,30$ & 0,11 & $-0,48^{*}$ \\
\hline Cadre & $-0,03$ & $0,99 * *$ & 0,005 & 0,41 & 0,16 & 0,06 \\
\hline $\begin{array}{l}\text { Profession } \\
\text { intermédiaire }\end{array}$ & 0,17 & $0,49 * *$ & 0,17 & 0,41 ** & 0,09 & 0,26 \\
\hline Employée & $-0,03$ & $0,31 * * *$ & $-0,04$ & $0,23 *$ & 0,01 & 0,10 \\
\hline Ouvrière & $-0,07$ & 0,07 & $-0,43^{*}$ & $-0,12$ & $-0,56^{\star}$ & $-0,19$ \\
\hline \multicolumn{7}{|l|}{ Diplôme du père } \\
\hline CAP, BEP, BEPC (réf.) & 0 & 0 & 0 & 0 & 0 & 0 \\
\hline Aucun diplôme ou CEP & $-0,47 * * *$ & $-0,27 * *$ & $-0,28 * * *$ & $-0,28^{* *}$ & 0,15 & $-0,14$ \\
\hline Baccalauréat & $0,30 *$ & $0,30 *$ & 0,47 ** & 0,37 ** & 0,51 ** & $0,34^{*}$ \\
\hline $\mathrm{Bac}+2$ & 0,27 & $0,94 * * *$ & $0,67 * * *$ & $0,77 * * *$ & $0,93 * *$ & $0,54 * *$ \\
\hline Supérieur & $0,51 * *$ & $1,13 * * *$ & $1,05^{* * *}$ & $1,00 * * *$ & $1,48 * * *$ & $0,77 * * *$ \\
\hline \multicolumn{7}{|l|}{ Diplôme de la mère } \\
\hline CAP, BEP, BEPC (réf.) & 0 & 0 & 0 & 0 & 0 & 0 \\
\hline Aucun diplôme ou CEP & $-0,49 * * *$ & $-0,56 * * *$ & $-0,48^{* * *}$ & $-0,49 * * *$ & $-0,12$ & $-0,26^{*}$ \\
\hline Baccalauréat & $0,57 * * *$ & $0,52 * * *$ & $0,44^{* * *}$ & $0,42 * * *$ & 0,15 & 0,25 \\
\hline $\mathrm{Bac}+2$ & $0,46^{*}$ & $0,42 *$ & $0,49 * * *$ & $0,72 * * *$ & 0,41 & $0,77 * * *$ \\
\hline Supérieur & $0,77^{* *}$ & 0,24 & 0,74 *** & 0,68 *** & 0,28 & $0,84^{* *}$ \\
\hline \multicolumn{7}{|l|}{ Sexe } \\
\hline Hommes (réf.) & 0 & 0 & 0 & 0 & 0 & 0 \\
\hline Femmes & $0,35 * * *$ & $0,58 * * *$ & $0,66 * * *$ & $0,67 * * *$ & $0,71 * * *$ & $0,51 * * *$ \\
\hline Constante & $-0,78 * * *$ & $-0,06$ & $-1,98 * * *$ & $-1,48 * * *$ & $-0,54 * *$ & $-0,52 * * *$ \\
\hline $\mathrm{G} 2$ & 6706 & 4309 & 5161 & 4113 & 2312 & 2887 \\
\hline Effectif & 5984 & 3889 & 5984 & 3889 & 2111 & 2454 \\
\hline \multicolumn{7}{|c|}{$\begin{array}{l}\text { Seuils de signification: }{ }^{*}: \mathrm{p} \leq 0,05 ;{ }^{* *}: \mathrm{p} \leq 0,01 ;{ }^{* * *}: \mathrm{p} \leq 0,001 \\
\text { Lecture : Le coefficient estimé pour les individus définis par la modalité active indique l'influence de cette } \\
\text { modalité, toutes choses étant égales par ailleurs, par rapport aux individus définis par la modalité de référence. } \\
\text { Le G2 (log-vraisemblance) permet d'estimer le pouvoir explicatif du modèle. } \\
\text { Source : calculs des auteurs à partir de l'enquête FQP } 2003 \text {. }\end{array}$} \\
\hline
\end{tabular}


Mais la baisse tendancielle de l'influence de l'origine socioprofessionnelle se conjugue avec une croissance du rôle du niveau d'instruction des parents, d'autant plus forte qu'il devient plus rare de ne pas accéder à ce niveau du baccalauréat.

Notons enfin que la diffusion du baccalauréat ne réduit pas l'avantage des filles, au contraire ${ }^{(8)}$. Comme le montrent les régressions, les filles ont surtout profité de la diversification des baccalauréats puisque leur avantage s'est réduit pour le seul baccalauréat général tout en s'accroissant globalement (toutes filières confondues).

\section{Quel baccalauréat?}

Examinons à présent le type de baccalauréat obtenu, dans la mesure où il conditionne fortement à la fois les taux de passage dans le supérieur, les orientations possibles et les taux de réussite ultérieure. Par rapport aux autres séries, l'obtention d'un baccalauréat général demeure plus inégalitaire ${ }^{(9)}$ (tableau 2), mais les inégalités baissent tout en restant à un niveau élevé. Entre les deux cohortes, les odds ratios passent de 14,3 à 11,2 : il y a donc une légère démocratisation en ce qui concerne l'obtention d'un baccalauréat général même si les différences de pourcentage s'accroissent légèrement. Ceci s'explique par le fait que le taux d'accès des enfants de cadres, même s'il augmente plus fortement en pourcentage que celui des enfants d'ouvriers, ne peut doubler, car il se situe déjà à un niveau élevé. De plus, l'augmentation de l'accès au baccalauréat des enfants de cadres a porté uniquement sur les séries générales alors que pour les enfants d'ouvriers, elle s'est répartie sur l'ensemble des types de bac, notamment le baccalauréat professionnel.

Les régressions logistiques (tableau 3) comparant les deux cohortes confirment cette évolution. L'influence de la profession du père sur la probabilité d'obtenir un baccalauréat général apparaît en baisse, alors que pour l'ensemble des autres paramètres pris en compte (profession de la mère, diplôme des deux parents, sexe), c'est la stabilité qui domine.

Enfin, en ce qui concerne l'obtention d'un baccalauréat technologique ou professionnel, les choses sont très différentes. Depuis leur création, ils ont toujours été plus populaires que les baccalauréats généraux. Comme le montrent les odds ratios et plus encore les différences de pourcentage (tableau 2), ils sont en passe de devenir de plus en plus populaires, au fur et à mesure que progresse l'accès au baccalauréat (dans la seconde cohorte). Ceci peut être mis en rapport avec l'observation précédente : dès lors que le baccalauréat général se diffuse (et donc que s'ouvre l'accès à un second cycle long), les élèves orientés

(8) Dans ce texte, nous ne traitons que de manière incidente des inégalités entre garçons et filles; sur ce point, on pourra se reporter à Duru-Bellat et al., 2001.

(9) Ceci renvoie aux mécanismes de l'orientation : celle-ci se fonde sur la valeur scolaire (les élèves les meilleurs allant vers un baccalauréat général), socialement inégale à ce stade, ainsi que sur les demandes des familles, marquées par une auto-sélection socialement différenciée, qui vient redoubler les inégalités de réussite (à réussite identique, les élèves de milieu aisé demandant davantage un baccalauréat général). 
en fin de $3^{e}$ vers les filières professionnelles (BEP) qui alimentent ensuite le baccalauréat du même nom sont issus de plus en plus des milieux populaires, puisque des inégalités sociales de réussite subsistent. En l'occurrence, le constat de démocratisation ségrégative s'impose.

\section{Parmi les bacheliers, avoir tel baccalauréat plutôt que tel autre}

Les constats précédents, établis sur l'ensemble de la population, mêlent deux phénomènes : l'ouverture de l'enseignement secondaire, d'une part, d'éventuelles modifications des orientations en son sein, d'autre part. Pour les dissocier, il convient de travailler spécifiquement sur la population bachelière. Comme le montre le tableau 4, les enfants d'ouvriers, quand ils atteignent ce niveau, ont une probabilité plus forte d'avoir un baccalauréat professionnel dans la seconde cohorte ; ils ont également moins de chances d'avoir accès à un baccalauréat général et à un baccalauréat technologique, alors que la distribution des types de baccalauréat pour les enfants de cadres n'a pratiquement pas changé. Les odds ratios traduisent bien ces différences d'évolution : pour ce qui est de l'accès à un baccalauréat général, ils sont croissants (de 4,7 à 6,2) tout comme les différences de pourcentages (qui passent de 33 à 39 points). Il y a donc bien une accentuation des inégalités sociales d'accès à la filière générale parmi les bacheliers. Pourtant, si on isole les chances d'accès à un baccalauréat technologique, on assiste à une très légère atténuation des inégalités sociales (les différences de pourcentage passent de -30 points à -21 points). C'est donc essentiellement le développement du baccalauréat professionnel - qui offre une chance d'accéder au niveau baccalauréat après un cycle professionnel court (CAP-BEP) - qui explique le renforcement des inégalités d'accès au baccalauréat général, dès lors que les enfants d'ouvriers forment l'essentiel de son « vivier».

\section{Tableau 4. Répartition des titulaires du baccalauréat selon le type de baccalauréat (en \%)}

\begin{tabular}{|c|c|c|c|c|c|}
\hline & \multicolumn{3}{|c|}{$\begin{array}{l}\text { Proportion de bacheliers } \\
\qquad(\text { en } \%)\end{array}$} & \multicolumn{2}{|c|}{$\begin{array}{c}\text { Écart entre enfants } \\
\text { de cadres et enfants } \\
\text { d'ouvriers }\end{array}$} \\
\hline & Tous & $\begin{array}{l}\text { Enfants } \\
\text { de cadres }\end{array}$ & $\begin{array}{c}\text { Enfants } \\
\text { d'ouvriers }\end{array}$ & $\begin{array}{l}\text { Différences de } \\
\text { pourcentage } \\
\text { (en points) }\end{array}$ & $\begin{array}{l}\text { Odds } \\
\text { ratios }\end{array}$ \\
\hline \multicolumn{6}{|l|}{ Cohorte 1962-1967 } \\
\hline Baccalauréat général & 63 & 82 & 49 & 33 & 4,7 \\
\hline Baccalauréat technologique & 30 & 13 & 43 & -30 & 0,2 \\
\hline Baccalauréat professionnel & 7 & 5 & 8 & -3 & 0,6 \\
\hline \multicolumn{6}{|l|}{ Cohorte 1975-1980 } \\
\hline Baccalauréat général & 62 & 83 & 44 & 39 & 6,2 \\
\hline Baccalauréat technologique & 23 & 11 & 32 & -21 & 0,3 \\
\hline Baccalauréat professionnel & 15 & 6 & 24 & -18 & 0,2 \\
\hline
\end{tabular}


Le modèle expliquant la probabilité d'obtenir un baccalauréat général dans la seule population bachelière ( $3^{\mathrm{e}}$ modèle du tableau 3 ) confirme que l'accès à ce diplôme ne s'est pas démocratisé ; au contraire, l'influence de la profession du père s'est accrue, légèrement pour les enfants de cadres et plus nettement pour les enfants d'indépendants (dans un sens positif), alors que les enfants d'employés tendent au contraire à se rapprocher de ceux des ouvriers; l'influence positive du niveau d'instruction de la mère s'est également accentuée, particulièrement pour les niveaux les plus élevés, au fur et à mesure que l'accès au baccalauréat se généralisait jusqu'à concerner une majorité de la classe d'âge : accéder ou non au baccalauréat général devient alors de plus en plus lié au niveau de diplôme de la mère, même si on remarque que les coefficients exprimant l'influence du niveau de diplôme paternel sont, quant à eux, en baisse.

Il est donc fondé de parler de démocratisation ségrégative : la démocratisation est advenue principalement du fait de la diffusion des baccalauréats professionnels, alors que dans le même temps, le fait d'obtenir un baccalauréat général devenait relativement de plus en plus difficile pour les enfants des familles les moins instruites.

Le type de baccalauréat est donc devenu en quelques décennies un vecteur important des inégalités sociales : les élèves sont maintenus plus longtemps dans le système scolaire, mais ils sont orientés dans des filières clairement hiérarchisées. Dès lors qu'une part importante d'une génération parvient au baccalauréat, cette population va se différencier selon des critères plus fins. L'examen des inégalités sociales doit donc concerner, outre le type de baccalauréat, la série du baccalauréat et le brio avec lequel il a été obtenu.

\section{Obtenir un baccalauréat scientifique, obtenir une mention}

Il est pertinent dans cette perspective de s'intéresser tout particulièrement au baccalauréat scientifique, qui est aujourd'hui le plus prestigieux et qui offre le plus de débouchés. Dans l'ensemble de la population, l'obtention d'un baccalauréat scientifique s'est démocratisée ${ }^{(10)}$ : les odds ratios passent de 15,9 à 6,4 entre les deux cohortes. Les inégalités sociales par rapport à cette série sont nettement plus faibles que pour le baccalauréat général et le mouvement de démocratisation y est nettement plus marqué, alors qu'on aurait pu penser que l'accès à cette série prestigieuse allait rester relativement protégé de l'ouverture générale. Cette démocratisation égalisatrice est confirmée par le premier modèle du tableau 5 dans lequel les coefficients exprimant l'influence de la catégorie socioprofessionnelle du père sont en baisse.

(10) Les séries scientifiques du baccalauréat sont regroupées dans l'enquête FQP. Pour la cohorte 1962-1967, sont regroupées les séries C (mathématiques), D (sciences expérimentales) et E (mathématiques et technologies); pour la cohorte 1975-1980, concernée par la réforme du baccalauréat de 1993, toutes les séries scientifiques sont incluses dans la série S, avec différentes spécialités, ici également regroupées. Cette réforme n’a guère affecté les caractéristiques scolaires et sociales des publics concernés (Convert, 2006). 


\section{Tableau 5. Évolution de l'influence des caractéristiques individuelles sur les chances d'obtention d'un baccalauréat scientifique (coefficients des modèles logit)}

\begin{tabular}{|c|c|c|c|c|c|c|}
\hline & \multicolumn{2}{|c|}{$\begin{array}{l}\text { Modèle } 1 \\
\text { Ensemble } \\
\text { de la cohorte }\end{array}$} & \multicolumn{2}{|c|}{$\begin{array}{l}\text { Modèle } 2 \\
\text { Bacheliers }\end{array}$} & \multicolumn{2}{|c|}{$\begin{array}{l}\text { Modèle } 3 \\
\text { Bacheliers } \\
\text { généraux }\end{array}$} \\
\hline & $\begin{array}{l}\text { Cohorte } \\
1962-1967\end{array}$ & $\begin{array}{l}\text { Cohorte } \\
1975-1980\end{array}$ & $\begin{array}{l}\text { Cohorte } \\
\text { 1962-1967 }\end{array}$ & $\begin{array}{l}\text { Cohorte } \\
1975-1980\end{array}$ & $\begin{array}{l}\text { Cohorte } \\
1962-1967\end{array}$ & $\begin{array}{l}\text { Cohorte } \\
1975-1980\end{array}$ \\
\hline \multicolumn{7}{|l|}{ Profession du père } \\
\hline Ouvrier (réf.) & 0 & 0 & 0 & 0 & 0 & 0 \\
\hline Agriculteur & $0,56^{*}$ & $0,80 * *$ & 0,112 & $0,94 * *$ & 0,45 & 0,97 * \\
\hline $\begin{array}{l}\text { Artisan, commerçant, } \\
\text { chef d'entreprise }\end{array}$ & $0,93 * * *$ & $0,70 * * *$ & $0,40^{*}$ & $0,43^{*}$ & 0,34 & $-0,12$ \\
\hline Cadre & $1,36^{* * *}$ & $0,77 * * *$ & $0,59 * *$ & $0,50 * *$ & 0,31 & $-0,006$ \\
\hline Profession intermédiaire & $-1,03 * * *$ & $0,48 * *$ & $0,41 *$ & 0,20 & 0,16 & $-0,18$ \\
\hline Employé & $0,84 * * *$ & 0,15 & $0,58^{* *}$ & $-0,002$ & 0,24 & $-0,33$ \\
\hline Autre & 0,56 & $-0,48$ & 0,40 & $-0,37$ & $-0,05$ & 0,05 \\
\hline \multicolumn{7}{|l|}{ Profession de la mère } \\
\hline Inactive (réf.) & 0 & 0 & 0 & 0 & 0 & 0 \\
\hline Agricultrice & 0,42 & $-0,36$ & 0,53 & $-0,88$ & 0,81 & $-0,95$ \\
\hline $\begin{array}{l}\text { Artisane, commerçante, } \\
\text { chef d'entrprise }\end{array}$ & 0,11 & $-0,32$ & 0,11 & $-0,40$ & 0,10 & $-0,20$ \\
\hline Cadre & 0,16 & 0,32 & 0,20 & 0,19 & 0,15 & 0,25 \\
\hline Profession intermédiaire & 0,19 & $0,33^{*}$ & 0,12 & 0,25 & 0,11 & 0,20 \\
\hline Employée & $-0,09$ & 0,09 & $-0,05$ & 0,003 & $-0,09$ & $-0,11$ \\
\hline Ouvrière & $-0,35$ & $-0,17$ & $-0,23$ & $-0,19$ & 0,07 & $-0,19$ \\
\hline \multicolumn{7}{|l|}{ Diplôme du père } \\
\hline CAP, BEP, BEPC (réf.) & 0 & 0 & 0 & 0 & 0 & 0 \\
\hline Aucun diplôme ou CEP & $-0,30 *$ & $-0,43 * *$ & $-0,03$ & $-0,34$ & $-0,18$ & $-0,15^{* *}$ \\
\hline Baccalauréat & $0,54 * *$ & 0,18 & 0,48 * & 0,09 & 0,28 & $-0,17$ \\
\hline $\mathrm{Bac}+2$ & $0,80 * * *$ & $0,54 * *$ & $0,85^{* * *}$ & $0,33^{*}$ & $0,56^{*}$ & 0,03 \\
\hline Supérieur & $1,08 * * *$ & $0,70 * * *$ & $1,06 * * *$ & $0,50 * *$ & $0,56^{*}$ & 0,17 \\
\hline \multicolumn{7}{|l|}{ Diplôme de la mère } \\
\hline CAP, BEP, BEPC (réf.) & 0 & 0 & 0 & 0 & 0 & 0 \\
\hline Aucun diplôme ou CEP & $-0,38 * *$ & $-0,52 * * *$ & $-0,10$ & $-0,29 *$ & 0,02 & $-0,12$ \\
\hline Baccalauréat & 0,29 & $0,28 *$ & 0,07 & 0,15 & $-0,003$ & 0,02 \\
\hline $\mathrm{Bac}+2$ & $0,48^{*}$ & $0,36^{*}$ & 0,34 & 0,28 & 0,21 & $-0,03$ \\
\hline Supérieur & $0,73 * *$ & 0,10 & 0,61 * & 0,01 & 0,55 & $-0,44$ \\
\hline \multicolumn{7}{|l|}{ Sexe } \\
\hline Homme (réf.) & 0 & 0 & 0 & 0 & 0 & 0 \\
\hline Femme & $-0,16$ & $-0,41 * * *$ & $-0,44^{* * *}$ & $-0,65^{* * *}$ & $-1,12^{* * *}$ & $-1,29 * * *$ \\
\hline Constante & $-2,69 * * *$ & $-1,68^{* * *}$ & $-1,30 * * *$ & $-0,82 * * *$ & 0,20 & $0,95^{* * *}$ \\
\hline G2 & 3348 & 3182 & 2349 & 2692 & 1646 & 1860 \\
\hline Effectif & 5984 & 3889 & 2111 & 2454 & 1306 & 1465 \\
\hline \multicolumn{7}{|c|}{$\begin{array}{l}\text { Seuils de signification: }{ }^{*}: p \leq 0,05 ; * *: p \leq 0,01 ;{ }^{* *}: p \leq 0,001 \text {. } \\
\text { Lecture : Le coefficient estimé pour les individus définis par la modalité active indique l'influence de cette } \\
\text { modalité, toutes choses étant égales par ailleurs, par rapport aux individus définis par la modalité de référence. } \\
\text { Le G2 (log-vraisemblance) permet d'estimer le pouvoir explicatif du modèle. } \\
\text { Source : calculs des auteurs à partir de l'enquête FQP } 2003 \text {. }\end{array}$} \\
\hline
\end{tabular}


Dans la population bachelière, le poids du baccalauréat scientifique est stable puisque 30,6 \% des bacheliers en sont dotés dans la première cohorte, et 29,4\% dans la seconde. Dans le contexte général d'expansion qu'on a connu, la part relative des séries scientifiques n’a donc pas augmenté. Pour autant, on observe une certaine démocratisation : $48,4 \%$ des enfants de cadres obtenaient un baccalauréat scientifique dans la première cohorte, contre 16,7 \% des enfants d'ouvriers. Dans la seconde, les chiffres correspondants sont de 44,2 \% et de $19,7 \%$. Les odds ratios traduisent cette évolution, puisqu'ils passent de 4,7 à 3,2. Le deuxième modèle du tableau 5 montre une atténuation de l'influence de la profession du père ainsi que de son niveau d'instruction (aucune des caractéristiques maternelles n'étant significative dans les deux cohortes).

Si l'on restreint l'analyse aux seuls bacheliers généraux, les chiffres montrent à nouveau un net resserrement entre les groupes sociaux. Dans la première cohorte, $59 \%$ des enfants de cadres obtiennent un baccalauréat scientifique contre $35 \%$ des enfants d'ouvriers. Dans la seconde cohorte, les chiffres correspondants sont de $53 \%$ pour les enfants de cadres et de $45 \%$ pour les enfants d'ouvriers. La lecture des odds ratios conforte ce constat d'une baisse de l'inégalité : ils passent de 2,7 à 1,2. Les modèles expliquant l'obtention d'un baccalauréat scientifique plutôt qu'un autre, sur la seule population qui obtient un baccalauréat général (cf. troisième modèle du tableau 5) montrent qu'à ce niveau, il n'y a plus d'influence significative de l'origine sociale; le fait d'avoir un père diplômé du supérieur qui jouait un rôle significatif dans la première cohorte ne l'est plus dans la seconde. Les enfants de milieu populaire - ceci vaut plus encore pour les enfants d'agriculteurs, du moins les meilleurs d'entre eux, ceux qui ne sont pas orientés vers le baccalauréat professionnel - ont profité de l'accès à un baccalauréat général pour s'orienter vers un baccalauréat scientifique, soit parce qu'ils sont particulièrement bons (ayant été relativement sur-sélectionnés concernant l'orientation vers un baccalauréat général), soit qu'il leur apparaisse plus attractif dans son contenu ou dans ses débouchés ${ }^{(11)}$. Réciproquement, les nouveaux bacheliers enfants de cadres, sans doute plus médiocres sur le plan scolaire (puisque jusqu'alors non-bacheliers, dans un groupe social où c'était la règle), se sont orientés vers d'autres séries que la série scientifique. On ne peut donc, quand on se situe à ce niveau de sélection scolaire que constitue le fait d'avoir accès à un baccalauréat général, parler de démocratisation ségrégative : il faut réserver ce terme à ce qui se joue dans l'accès à tel ou tel type de bac. Au contraire, on tendrait à ce stade vers plus de méritocratie.

Il conviendrait bien sûr de nuancer ce constat en prenant en compte les options qui existent au sein de toutes les séries et en l'occurrence au sein de la série scientifique. Ceci ne peut être fait à partir des données de l'enquête FQP ; rappelons seulement que les données de la direction de l'Évaluation, de 
la Prospective et de la Performance (DEPP) du ministère de l'Éducation nationale montrent par exemple que, dans le panel d'élèves entrés en $6^{\mathrm{e}}$ en 1995 , $28,5 \%$ des enfants de cadres ont obtenu un baccalauréat scientifique avec l'option mathématiques et physique, ce qui n'est le cas que de 4,6\% des enfants d'ouvriers (l'odds ratio correspondant étant de 8,2).

Pour ce qui est du baccalauréat scientifique, les filles et les garçons se différencient, puisque les filles accèdent significativement moins souvent à cette série. Ceci vaut à la fois quand on prend en compte l'ensemble de la population (de manière assez faible, puisque par ailleurs les filles accèdent plus souvent à un baccalauréat) et l'ensemble des bacheliers, et plus encore pour la seule population obtenant un baccalauréat général (c'est le seul facteur qui joue fortement et significativement parmi les bacheliers généraux). Aux trois niveaux d'analyse, ce phénomène tend plutôt à s'accentuer entre les deux cohortes.

Enfin, un autre indicateur de l'excellence est pertinent : l'obtention d'une mention, c'est-à-dire d'une moyenne d'au moins 12 sur 20 aux épreuves du baccalauréat. Ceci joue en particulier pour l'accès à certaines filières sélectives de l'enseignement supérieur. La probabilité d'avoir une mention est plus élevée pour les baccalauréats généraux (respectivement $29,5 \%$ et 35,3\% dans la première et la seconde cohorte) que pour les baccalauréats technologiques (13\% et 25,7 \%), mais elle est particulièrement élevée pour les baccalauréats professionnels (19,6\% et $41,8 \%$ ). Pour ce qui concerne les mentions les plus élevées (« bien » ou « très bien » soit respectivement au moins 14 et 16 de moyenne), celles qui favorisent le plus l'accès aux filières d'« élite » les plus prestigieuses, l'avantage reste nettement aux bacheliers généraux $(13,6 \%$, contre moins de $5 \%$ pour les bacheliers technologiques et 9,8\% pour les bacheliers professionnels dans la seconde cohorte). L'obtention d'une mention est plus fréquente pour les enfants de cadres, ce qui rejoint les constats antérieurs faits par Convert et Pinet (1989) : parmi les bacheliers généraux de la seconde cohorte, $41,3 \%$ des enfants de cadres ont obtenu une mention, contre $29,4 \%$ des enfants d'ouvriers. Néanmoins, à ce stade de la sélection, les inégalités sociales deviennent beaucoup moins marquées, comme le montre le tableau 6 .

Tableau 6. Inégalités entre enfants de cadres et enfants d'ouvriers à différents niveaux d'excellence (odds ratios)

\begin{tabular}{|c|c|c|c|c|}
\hline & $\begin{array}{c}\text { Obtention du } \\
\text { baccalauréat } \\
\text { dans l'ensemble } \\
\text { de la } \\
\text { population }\end{array}$ & $\begin{array}{c}\text { Obtention du } \\
\text { baccalauréat } \\
\text { général parmi } \\
\text { les titulaires } \\
\text { d'un } \\
\text { baccalauréat }\end{array}$ & $\begin{array}{c}\text { Obtention } \\
\text { d'un bac } \\
\text { scientifique } \\
\text { parmi les } \\
\text { titulaires d'un } \\
\text { bac général }\end{array}$ & $\begin{array}{c}\text { Obtention } \\
\text { d'une mention } \\
\text { parmi les } \\
\text { titulaires d'un } \\
\text { baccalauréat } \\
\text { scientifique }\end{array}$ \\
\hline $\begin{array}{c}\text { Cohorte 1962-1967 } \\
\text { Cohorte 1975-1980 }\end{array}$ & 11,4 & 4,7 & 2,7 & 1,3 \\
\hline
\end{tabular}

Source : calculs des auteurs d'après l'enquête FQP 2003. 
La population qui sera in fine éligible pour l'accès à l'enseignement supérieur est donc progressivement stratifiée selon le type et la série du baccalauréat ainsi que la mention obtenue. Cette segmentation est à la fois scolaire et sociale. Le tableau 6 montre bien qu'une part importante de la sélection sociale se fait en amont de l'accès à un baccalauréat, par le jeu conjugué des inégalités de réussite et des inégalités d'orientation. Parmi cette population qui devient bachelière, les inégalités sont déjà un peu moins fortes en ce qui concerne les chances d'obtenir un baccalauréat général. Parmi les titulaires d'un bac de ce type, tout se passe comme si les inégalités sociales s'estompaient cette fois devant des inégalités proprement scolaires : degré inégal de réussite appréhendé par le choix d'une série scientifique, puis obtention d'une mention au sein de cette série.

Que peut-on attendre de ces évolutions quant aux inégalités d'accès et d'orientation dans l'enseignement supérieur ? Dès lors que l'obtention du baccalauréat s'avère moins sélective, on peut s'attendre (comme maints auteurs l'ont souligné, par exemple Mare, 1981) à ce qu'une population plus large et plus hétérogène se présente à ce nouveau palier d'orientation et que les inégalités sociales qui s'étaient estompées au stade antérieur s'accroissent à ce niveau, soit en ce qui concerne les taux globaux d'accès, soit en ce qui concerne les trajectoires au sein de ce niveau d'enseignement.

\section{L'évolution des transitions entre le secondaire et le supérieur}

Le baccalauréat étant un passeport pour l'enseignement supérieur, stade ultime de la scolarité, la question de la démocratisation se déplace à présent à ce dernier niveau d'enseignement. Dans un premier temps, nous allons décrire les changements observés entre les deux groupes de cohortes, en raisonnant sur l'ensemble de la population, c'est-à-dire en prenant en compte l'ouverture du système en amont et la démocratisation des carrières scolaires qui en a résulté aux niveaux antérieurs. Dans un second temps, nous nous focaliserons sur les évolutions au sein de la population des bacheliers.

\section{Les chances d'entrer dans l'enseignement supérieur et d'obtenir un diplôme}

Commençons par l'examen des taux d'accès à l'enseignement supérieur pour l'ensemble de la population. Ceux-ci ont très fortement augmenté dans le bref laps de temps qui sépare les deux cohortes (de 27,7 \% d'une génération à $53,2 \%$ ). Pourtant, les inégalités sociales ont peu évolué. Tous les groupes sociaux ayant bénéficié de manière comparable de cette expansion, les différences de pourcentage et les odds ratios (tableau 7) évoluent certes à la baisse, mais très faiblement (de 11,4 à 11,2). On est dans le cas de figure que P. Merle (2000) qualifie de « démocratisation uniforme », où se conjuguent taux d'accès 
en hausse et inégalités stables. L'ouverture très nette de l'accès à l'enseignement supérieur n'aurait donc pas contribué à réduire les inégalités sociales, sans doute parce qu'on reste loin des « effets de plafond». Cette situation est néanmoins amenée à évoluer très vite puisque le taux d'accès des enfants de cadres dépasse $85 \%$ dans la seconde cohorte.

Tableau 7. Évolution des inégalités d'accès à l'enseignement supérieur

\begin{tabular}{|c|c|c|c|c|c|}
\hline & \multicolumn{3}{|c|}{ Taux d'accès (en \%) } & \multicolumn{2}{|c|}{$\begin{array}{c}\text { Écart entre enfants } \\
\text { de cadres et enfants } \\
\text { d'ouvriers }\end{array}$} \\
\hline & Tous & $\begin{array}{l}\text { Enfants } \\
\text { de cadres }\end{array}$ & $\begin{array}{c}\text { Enfants } \\
\text { d'ouvriers }\end{array}$ & $\begin{array}{l}\text { Différences de } \\
\text { pourcentage } \\
\text { (en points) }\end{array}$ & $\begin{array}{l}\text { Odds } \\
\text { ratios }\end{array}$ \\
\hline \multicolumn{6}{|c|}{ Ensemble de la cohorte } \\
\hline Cohorte 1962-1967 & 27,7 & 66,1 & 14,6 & 51,5 & 11,4 \\
\hline Cohorte 1975-1980 & 53,2 & 85,3 & 34,2 & 51,1 & 11,2 \\
\hline \multicolumn{6}{|l|}{ Parmi les bacheliers } \\
\hline Cohorte 1962-1967 & 75,3 & 85,1 & 67,7 & 17,4 & 2,7 \\
\hline Cohorte 1975-1980 & 81,7 & 92,9 & 70,7 & 22,2 & 5,4 \\
\hline \multicolumn{6}{|c|}{ Parmi les bacheliers généraux } \\
\hline Cohorte 1962-1967 & 88,6 & 92,6 & 84,3 & 8,3 & 2,3 \\
\hline Cohorte 1975-1980 & 95,4 & 95,9 & 93,0 & 2,9 & 1,8 \\
\hline \multicolumn{6}{|c|}{ Parmi les bacheliers technologiques et professionnels } \\
\hline Cohorte 1962-1967 & 52,8 & 51,6 & 52,6 & $-1,0$ & 1,0 \\
\hline Cohorte 1975-1980 & 60,4 & 77,8 & 53,6 & 24,2 & 3,0 \\
\hline
\end{tabular}

Cette stabilité est relativement surprenante puisqu'il y a eu une démocratisation sensible de l'obtention du baccalauréat dans l'ensemble de la population et que par ailleurs, les bacheliers sont très nombreux à accéder à l'enseignement supérieur. Mais la population des bacheliers est elle-même très hétérogène. Les taux d'accès au supérieur sont effectivement proches de $100 \%$ pour les élèves dotés d'un baccalauréat général : dès la première cohorte, $88,6 \%$ d'entre eux ont accédé à l'enseignement supérieur, et c'est le cas de 95,4\% dans la seconde cohorte. En ce qui concerne les bacheliers technologiques, les taux sont moindres, même s'ils ont augmenté, de $56 \%$ dans la première cohorte à $78 \%$ dans la seconde. Enfin, concernant les bacheliers professionnels, les effectifs sont faibles en début de période, le diplôme ayant été créé en 1987 ; on ne peut donc estimer avec une grande fiabilité l'évolution entre les deux cohortes (les taux d'accès passent de 3,5 à $6 \%)^{(12)}$.

(12) Ces dernières années, les taux de poursuite d'études supérieures parmi les bacheliers professionnels sont passés de $17,3 \%$ en 1999 à $28,3 \%$ en 2004, dont $58,3 \%$ dans les filières professionnelles courtes. Notons que ces étudiants rencontrent des difficultés pour être admis dans les filières professionnelles sélectives par rapport aux bacheliers technologiques et généraux; ils se 
Pour comprendre la coexistence d'une démocratisation de l'obtention du baccalauréat d'un côté, et de la très faible évolution des inégalités d'accès au supérieur de l'autre, il faut prendre en compte l'hétérogénéité de la population bachelière et s'intéresser spécifiquement aux taux de passage et aux orientations des différents types de bacheliers.

Sur l'ensemble des bacheliers, les taux d'accès à l'enseignement supérieur des enfants de cadres ont augmenté (ils atteignent presque $93 \%$ dans la seconde cohorte) alors que la croissance a été faible pour les enfants d'ouvriers. La progression globale de l'accès à l'enseignement supérieur, pour les bacheliers, ne s'accompagne pas d'une réduction des écarts, au contraire : les différences de pourcentage s'accroissent et les odds ratios plus encore. Ils atteignent 5,4 en fin de période (contre, rappelons-le, 11,2 pour l'ensemble de la population) ${ }^{(13)}$. Sur la seule population des bacheliers (tous types de baccalauréats réunis), c'est donc à une augmentation des inégalités sociales d'accès à l'enseignement supérieur que l'on assiste. Tout se passe comme si les inégalités qui s'étaient estompées pour l'accès au baccalauréat s'étaient accentuées une fois ce seuil franchi ; en d'autres termes, le titre de bachelier garantit de façon de moins en moins homogène socialement l'entrée dans l'enseignement supérieur, en raison de la diversification du diplôme lui-même, due à l'importance croissante des baccalauréats technologiques et professionnels.

Parmi les seuls bacheliers généraux, on observe une légère baisse des inégalités (mais presque tous poursuivent des études supérieures), qui apparaissent, à ce stade, relativement faibles. Le constat précédent ne s'explique donc pas par des inégalités sociales plus marquées au sein des différents types de bac, mais par l'évolution des inégalités qui affectent l'orientation entre ceux-ci, nous y reviendrons.

Enfin, lorsqu'on regroupe les bacheliers technologiques et les bacheliers professionnels, on constate une hausse des inégalités sociales en raison du développement du baccalauréat professionnel qui ouvre beaucoup moins l'accès à l'enseignement supérieur que le baccalauréat technologique et qui est par ailleurs encore plus populaire.

Qu'en est-il des chances d'obtenir un diplôme au terme du cursus universitaire, ce qui constitue l'enjeu essentiel dans la perspective de l'insertion professionnelle? À cet égard, les odds ratios entre enfants de cadres et enfants d'ouvriers sont en baisse entre les deux cohortes (1962-1967 et 1972-1977 ${ }^{(14)}$ ),

« rabattent » alors sur l'université, où leur taux d'échec est extrêmement élevé : ces dernières années, la probabilité d'obtenir un Deug (en deux ans ou plus) variait de $83 \%$ pour les bacheliers généraux et $40 \%$ pour les bacheliers technologiques à $15 \%$ seulement pour les bacheliers professionnels (Source : ministère de l'Éducation nationale, L'état de l'école, éd. 2005).

(13) Il n'est pas pertinent de chercher à modéliser le passage des bacheliers dans l'enseignement supérieur - et a fortiori des bacheliers généraux - dans la mesure où les taux de passage sont très proches de $100 \%$ : les individus qui s'arrêtent sont peu nombreux et peu différenciés socialement.

(14) Rappelons que les générations retenues sont ici décalées de 3 ans pour tenir compte de l'achèvement des études dans la seconde cohorte. 
de 10,8 dans le premier groupe à 9,2 dans le second. On retrouve donc cette tendance à la démocratisation, qui poursuit celle observée au niveau de diplôme précédent (celui du baccalauréat). Tout se passe comme si la «vague démocratisante » qui a démarré les années précédentes en France avec l'ouverture de l'accès au collège, puis au lycée (Duru-Bellat et Kieffer, 2000), venait d'atteindre le niveau du supérieur : les chances d'obtenir un diplôme du supérieur sont devenues moins inégales entre les groupes sociaux depuis les années 1980 et la prise en compte de cohortes encore plus récentes (les générations 19751980) montrerait que cette évolution se poursuit. Pour l'heure, il reste en fin de période des écarts de taux très importants, puisque seulement 17,2\% des enfants d'ouvriers de la seconde cohorte obtiennent un diplôme du supérieur, contre $65,6 \%$ des enfants de cadres.

Dans une seconde étape, travailler spécifiquement sur la seule population bachelière permet de démêler deux évolutions : l'évolution de l'accès au baccalauréat d'une part, l'évolution des cursus dans le supérieur d'autre part (soit le fait d'y accéder et le fait d'y obtenir un diplôme). Avec cette seconde perspective (obtention d'un diplôme chez les seuls bacheliers), les odds ratios sont trois fois moins importants, car la plus grande part des inégalités sociales jouent antérieurement, dans le fait d'accéder ou non à un baccalauréat. Surtout, les odds ratios augmentent légèrement entre les deux cohortes (de 2,8 à 3,3); ce léger accroissement s'explique par le constat précédent, à savoir l'augmentation des inégalités de passage dans l'enseignement supérieur parmi les bacheliers.

Une première conclusion est donc que la démocratisation de l'obtention d'un diplôme du supérieur, qui est réelle, résulte entièrement de la diffusion du titre qui rend éligible à ce niveau, le baccalauréat ; l'accès au supérieur, en lui-même, ne s'est pas démocratisé, du fait de l'évolution des inégalités d'obtention de tel ou tel type de bac, en d'autres termes du caractère ségrégatif de cette démocratisation.

Dans un troisième temps, intéressons-nous à l'évolution des inégalités pour la population la plus sélectionnée, les bacheliers généraux. Les inégalités sont plus faibles dans l'absolu et tendent à se réduire dans le temps : tant les différences de pourcentage que les odds ratios sont en très légère baisse avec en fin de période un chiffre de 2,1 ; on ne saurait donc dire que les inégalités quant aux chances d'obtenir un diplôme ont évolué significativement dans cette sous-population.

\section{Les étapes du processus sélectif jusqu'à un diplôme du supérieur}

L'ensemble de ces résultats soulignent la transformation des mécanismes d'inégalités sociales qui ont accompagné le développement de l'accès à l'éducation ces vingt dernières années. Il y a eu une ouverture très importante de l'accès d'une classe d'âge au baccalauréat, mais elle ne s'est pas traduite par une 
démocratisation proportionnelle de l'accès à l'enseignement supérieur parce que les enfants d'ouvriers n'ont vu augmenter leurs chances d'obtenir un baccalauréat qu'au prix d'une relégation de fait dans les filières technologiques et professionnelles. Ceci a entraîné une accentuation des inégalités au sein de la population bachelière, pour une double raison : d'abord parce que ces bacheliers ont moins souvent accès au supérieur, ensuite parce que leurs chances d'y réussir sont nettement plus faibles, compte tenu des processus d'orientation par l'échec (scolaire) qui y conduisent ${ }^{(15)}$. En revanche, ceux des enfants de milieu populaire qui ont eu accès aux baccalauréats généraux (en moindre proportion par rapport aux autres bacs) ont vu leurs chances de poursuivre des études supérieures se rapprocher (un peu) de celles des enfants de cadres.

De la même manière, on voit clairement que si globalement l'obtention d'un diplôme du supérieur s'est démocratisée, cette démocratisation est due entièrement à celle du baccalauréat. En effet, si l'on adopte une perspective conditionnelle, c'est-à-dire si l'on se focalise sur la seule population bachelière, aucune démocratisation n'est à l'œuvre ; au contraire, la tendance est plutôt à une légère hausse des inégalités. Cette légère hausse ne s'observe plus si on isole les jeunes bacheliers généraux. Par conséquent, le seul mécanisme qui explique la hausse des inégalités quant aux chances d'obtenir un diplôme du supérieur est bien l'orientation vers tel ou tel type de baccalauréat (avec les limitations éventuelles qui en résultent pour la suite des cursus). Avec le développement général de l'accès à l'éducation, les enfants de milieu populaire deviennent certes de plus en plus fréquemment bacheliers, mais ils sont plus fortement canalisés dans les voies technologiques et (plus encore) professionnelles, ce qui leur donne moins de chances d'entrer dans l'enseignement supérieur et de réussir à y obtenir un diplôme. En revanche, quand ces jeunes ont accès à un baccalauréat général, à nouveau, les inégalités sociales tendent plutôt à baisser légèrement et les modèles montrent qu'elles ne sont plus significatives.

Nos résultats diffèrent en partie de ceux obtenus par Selz et Vallet (2006) à partir des enquêtes Emploi, qui cherchent à expliquer les inégalités sur la longue période eu égard au plus haut diplôme obtenu. Ils observent dans un premier temps, sur l'ensemble des cohortes et pour des générations nées entre 1920 et 1976, une baisse des inégalités sociales, concentrée sur les générations 1938-1946, moins marquée ensuite. Certes, nos résultats portent sur une période plus limitée et plus récente et concernent uniquement l'obtention d'un diplôme du supérieur, mais la tendance lourde à une démocratisation est confirmée. Quand ces auteurs isolent la population bachelière, le constat se retourne et les inégalités apparaissent en hausse, ce qui concorde avec nos résultats. Par contre, ils obtiennent, sur la période qu'ils étudient, des inégalités en hausse sur la population des seuls bacheliers généraux, alors que nous concluons à une légère baisse des inégalités. Cela nous amène à interpréter les 
évolutions comme relevant avant tout des glissements qui se sont opérés entre les différents types de bac ; de leur côté, Selz et Vallet invoquent plutôt l'hétérogénéité croissante de la population bachelière, ce qui constitue plus une piste d'interprétation complémentaire que concurrente ${ }^{(16)}$.

Alors que la sélection sociale passait, jusqu'au milieu des années 1980 (avant l'explosion du taux d'accès au bac), par la réalisation d'une scolarité secondaire complète et le niveau de diplôme atteint à l'issue d'un parcours qui pouvait s'arrêter très vite, après les paliers d'orientation de $5^{\mathrm{e}}$ et de $3^{\mathrm{e}}$, elle réside à présent davantage dans le type de baccalauréat auquel on accède (général, technologique ou professionnel), même si bien évidemment la scolarité antérieure et les inégalités afférentes continuent d'exercer une influence, puisqu'elles pèsent sur les processus d'orientation entre ces différentes voies.

L'hypothèse de Arum et al. (2007), évoquée précédemment, est plutôt confortée par ces premiers résultats : du fait de la différenciation, qui n'est pas seulement « horizontale » mais "verticale», des différents types de bacs, les enfants de milieu populaire sont détournés des possibilités offertes par le baccalauréat général, ce qui, enseignement secondaire et enseignement supérieur étant étroitement articulés, a des incidences importantes sur les scolarités ultérieures. Ce phénomène de "diversion » rappelle certains constats établis après la création des baccalauréats technologiques à la fin des années 1960 par Cherkaoui (1982) montrant que ces nouveaux baccalauréats avaient de fait canalisé les enfants de milieu populaire dans ces filières, ce qui avait diminué leurs chances d'accéder aux baccalauréats généraux tels qu'ils existaient jusqu'alors.

Mais le type de baccalauréat obtenu et les contraintes qui en résultent quant aux scolarités ultérieures ne constituent pas la seule forme de différenciation des trajectoires. Il est pertinent, bien que cela soit rarement fait dans la littérature, d'étendre le questionnement aux types d'études et aux spécialités au sein de l'enseignement supérieur, ce que permettent les données des enquêtes FQP.

\section{L'évolution des choix au sein du supérieur et des inégalités sociales afférentes: une différenciation de plus en plus horizontale ?}

Pour analyser l'évolution des choix dans l'enseignement supérieur, une perspective légitime est de prendre en compte le premier choix, même si l'on sait non seulement que des réorientations suite à un échec se produiront, mais aussi que certains étudiants mettent en ouvre de véritables stratégies pour maximiser leurs chances d'atteindre tel ou tel diplôme « final », par exemple entrer en IUT

(16) Cette divergence peut être due aux sources mobilisées. Alors que les enquêtes Emploi recueillent les diplômes obtenus pour chaque membre du ménage auprès de la personne présente au moment de l'interrogation, les enquêtes FQP s'adressent à la personne elle-même. Ce dernier procédé est plus fiable, comme le montre la comparaison entre les réponses fournies à l'enquête Emploi et à une de ses enquêtes complémentaires passée la même année, la personne connaissant précisément son diplôme. 
(Institut universitaire de technologie) pour accéder ensuite à une école d'ingénieur ou une filière universitaire. En France, les jeunes peuvent théoriquement choisir n'importe quelle filière avec n'importe quel bac. Mais au-delà de ce principe très général, il existe à la fois des procédures explicites de sélection dans certaines filières - CPGE (classes préparatoires aux grandes écoles), IUT, STS (sections de techniciens supérieurs) notamment $-{ }^{(17)}$ et des processus d'autosélection de la part des étudiants, bien connus dans la littérature (Duru-Bellat, 2002). La possession d'un baccalauréat scientifique maximise les chances d'être admis dans les filières sélectives, même si des processus d'auto-sélection existent, y compris au sein des populations les plus sélectionnées scolairement, processus marqués notamment par l'origine sociale et le sexe de l'étudiant. Les analyses qui suivent prendront donc en compte ces différents paramètres.

\section{L'accès aux différents types d'études : le premier " grand choix " ?}

En France, on ne saurait traiter de l'enseignement supérieur comme d'un bloc indifférencié. Il existe des types de filières bien distinctes, qui ont inégalement la possibilité de sélectionner leurs étudiants, et qui sont diversement professionnalisées. Nous centrerons donc ici notre analyse, dans un premier temps, sur la dichotomie entre filières sélectives et filières non sélectives, en regroupant dans les filières sélectives dites d'« élite » les classes préparatoires, la médecine et les écoles d'ingénieurs qui recrutent directement au niveau du baccalauréat; nous les distinguerons de toutes les autres filières universitaires ouvertes (hors médecine), ainsi que des filières professionnelles courtes sélectives (IUT, STS, filières paramédicales et sociales, etc.).

Si on considère l'ensemble des cohortes pour appréhender les chances globales d'accéder à tel ou tel diplôme du supérieur, la démocratisation s'avère à l'œuvre dans toutes les filières puisque les odds ratios sont tous en baisse entre les deux groupes (tableau 8). Soulignons qu'on parvient au même constat si au lieu de travailler sur l'accès à l'enseignement supérieur, on travaille sur l'obtention du diplôme. On peut néanmoins noter combien l'accès aux filières d'« élite » reste, dans notre pays, profondément inégalitaire (avec un odds ratio de 12,1 dans la seconde cohorte) alors qu'on s'approche de la démocratisation pour les filières supérieures courtes.

(17) Les CPGE sont des formations de premier cycle de l'enseignement supérieur, dispensées au sein des lycées. Elles préparent en deux ans aux concours d'entrée dans les grandes écoles d'ingénieurs et de commerce-gestion. Ces filières très sélectives recrutent sur dossier des bacheliers (très majoritairement des bacheliers généraux) qui ont obtenu d'excellents résultats tout au long de l'année terminale.

Les STS sont des formations de premier cycle de l'enseignement supérieur qui préparent en deux ans, au sein des lycées, au brevet de technicien supérieur (BTS). Elles s'adressent en priorité aux élèves qui ont obtenu de bons résultats au baccalauréat technologique.

Les IUT sont des établissements de l'enseignement supérieur qui préparent en deux ans au diplôme universitaire de technologie (DUT). Cette filière sélective recrute sur dossier de bons élèves dotés du baccalauréat technologique ou du baccalauréat général (ces derniers utilisant souvent cette filière pour rejoindre ensuite des études longues, en école ou à l'université). 
Tableau 8. Évolution de l'accès des enfants d'ouvriers aux différents types d'études supérieures comparé à celui des enfants de cadres

\begin{tabular}{|c|c|c|c|c|c|}
\hline & \multicolumn{3}{|c|}{ Taux d'accès (en \%) } & \multicolumn{2}{|c|}{$\begin{array}{l}\text { Écart entre enfants } \\
\text { de cadres et enfants } \\
\text { d'ouvriers }\end{array}$} \\
\hline & Tous & $\begin{array}{l}\text { Enfants } \\
\text { de cadres }\end{array}$ & $\begin{array}{l}\text { Enfants } \\
\text { d'ouvriers }\end{array}$ & $\begin{array}{l}\text { Différences de } \\
\text { pourcentage } \\
\text { (en points) }\end{array}$ & $\begin{array}{l}\text { Odds } \\
\text { ratios }\end{array}$ \\
\hline \multicolumn{6}{|c|}{ Dans l'ensemble de la cohorte } \\
\hline \multicolumn{6}{|l|}{ Cohorte 1962-1967 } \\
\hline $\begin{array}{l}\text { Filières professionnelles } \\
\text { supérieures }\end{array}$ & 13,4 & 23,5 & 7,8 & 15,7 & 3,6 \\
\hline Université sauf médecine & 9,9 & 23,3 & 5,5 & 17,8 & 5,2 \\
\hline Filières d'élite & 4,4 & 19,3 & 1,3 & 18,0 & 18,2 \\
\hline \multicolumn{6}{|l|}{ Cohorte 1975-1980 } \\
\hline $\begin{array}{l}\text { Filières professionnelles } \\
\text { supérieures }\end{array}$ & 25,3 & 30,3 & 19,5 & 10,8 & 1,8 \\
\hline Université sauf médecine & 21,1 & 34,4 & 12,6 & 21,8 & 3,6 \\
\hline Filières d'élite & 6,9 & 20,6 & 2,1 & 18,5 & 12,1 \\
\hline \multicolumn{6}{|c|}{ Parmi les bacheliers } \\
\hline \multicolumn{6}{|l|}{ Cohorte 1962-1967 } \\
\hline $\begin{array}{l}\text { Filières professionnelles } \\
\text { supérieures }\end{array}$ & 35,8 & 16,6 & 23,0 & $-6,4$ & 0,7 \\
\hline Université sauf médecine & 27,3 & 22,3 & 21,6 & 0,7 & 1,0 \\
\hline Filières d'élite & 12,1 & 41,8 & 12,3 & 29,5 & 5,1 \\
\hline \multicolumn{6}{|l|}{ Cohorte 1975-1980 } \\
\hline $\begin{array}{l}\text { Filières professionnelles } \\
\text { supérieures }\end{array}$ & 38,3 & 17,2 & 27,9 & $-10,7$ & 0,5 \\
\hline Université sauf médecine & 32,6 & 23,9 & 21,5 & 2,4 & 1,1 \\
\hline Filières d'élite & 10,7 & 43,2 & 11,1 & 32,1 & 6,1 \\
\hline \multicolumn{6}{|c|}{ Parmi les bacheliers généraux } \\
\hline \multicolumn{6}{|l|}{ Cohorte 1962-1967 } \\
\hline $\begin{array}{l}\text { Filières professionnelles } \\
\text { supérieures }\end{array}$ & 34,0 & 21,5 & 18,4 & 3,1 & 1,2 \\
\hline Université sauf médecine & 36,5 & 24,6 & 18,3 & 6,3 & 1,5 \\
\hline Filières d'élite & 18,0 & 44,0 & 10,1 & 33,9 & 7,0 \\
\hline \multicolumn{6}{|l|}{ Cohorte 1975-1980 } \\
\hline $\begin{array}{l}\text { Filières professionnelles } \\
\text { supérieures }\end{array}$ & 33,2 & 23,8 & 21,4 & 2,4 & 1,1 \\
\hline Université sauf médecine & 46,1 & 25,1 & 19,4 & 5,7 & 1,4 \\
\hline Filières d'élite & 16,1 & 45,9 & 10,7 & 35,2 & 7,1 \\
\hline
\end{tabular}

Par contre, si on se centre sur les bacheliers, la conclusion est toute différente : on ne peut pas parler de démocratisation. Certes, les filières professionnelles courtes tendent à devenir de plus en plus populaires (ce qui est évidemment lié au fait que les enfants de milieu populaire ont de plus en plus de chances d'ob- 
tenir un baccalauréat technologique ou professionnel), mais les filières d'« élite » deviennent au contraire de plus en plus sélectives socialement, alors que la situation des filières universitaires est stable et l'égalité pratiquement réalisée. À nouveau, le terme de démocratisation ségrégative s'avère pertinent.

Ce constat n'est probablement pas sans rapport avec la stabilité du poids de ce secteur d'« élite». En effet, l'importance relative de l'ouverture des diverses filières, de même que l'importance relative de l'ouverture du niveau secondaire (définissant le volume des éligibles au niveau supérieur) sont des paramètres qui affectent l'évolution des inégalités sociales. Des études récentes (Albouy et Wanecq, 2003) ont montré que la démocratisation de l'ensemble du supérieur a épargné (en quelque sorte) les grandes écoles qui, dans un contexte d'expansion des effectifs, ont su maintenir à l'identique leur part relative. On relève même une accentuation des inégalités sociales d'accès aux écoles les plus prestigieuses.

Enfin, si on isole les seuls bacheliers généraux, un contraste très fort apparaît entre des orientations par rapport auxquelles l'égalité est pratiquement réalisée (les filières professionnelles courtes et, à un degré un peu moindre, les filières universitaires ouvertes) et des filières d'« élite» qui restent très fortement inégalitaires (avec un odds ratios de 7,1). Population sélectionnée, les bacheliers généraux entrent presque également (selon leur milieu social d'origine) dans l'enseignement supérieur, mais pour autant, les orientations restent inégales, du moins pour les filières d'« élite " qui résistent à toute évolution. Si entre les deux cohortes, les odds ratios évoluent extrêmement peu, ils évoluent plutôt à la baisse dans les filières professionnelles courtes et universitaires, et plutôt à la hausse dans les filières d'« élite ».

Il est utile de distinguer, dans cette inégalité d'accès aux filières d'« élite », le rôle de la valeur scolaire accumulée par les enfants de cadres et celui de choix d'orientation qui seraient significativement différents à valeur scolaire comparable. Pour éclairer cette question, nous avons estimé des modèles logit expliquant les premiers choix des étudiants (qui peuvent différer des diplômes obtenus in fine), en distinguant les types de filières. Dans la mesure où on peut considérer ces types comme ordonnés (en termes de hiérarchie scolaire et de débouchés), nous avons opposé dans un premier temps les filières d'« élite * à toutes les autres orientations puis, dans un second temps, en travaillant seulement sur les étudiants n'ayant pas accédé aux filières d'« élite », nous avons opposé les filières professionnelles courtes aux filières universitaires $\left(\right.$ tableau 9) ${ }^{(18)}$. 
Tableau 9. Choix des types d'études selon les caractéristiques individuelles (coefficients des modèles logit)

\begin{tabular}{|c|c|c|c|c|}
\hline & \multicolumn{2}{|c|}{$\begin{array}{l}\text { Modèle } 1 \\
\text { CPGE vs universités ou } \\
\text { filières professionnelles }\end{array}$} & \multicolumn{2}{|c|}{$\begin{array}{l}\text { Modèle } 2 \\
\text { Filières professionnelles } \\
\text { vs universités }\end{array}$} \\
\hline & $\begin{array}{c}\text { Cohorte } \\
1962-1967\end{array}$ & $\begin{array}{c}\text { Cohorte } \\
1975-1980\end{array}$ & $\begin{array}{c}\text { Cohorte } \\
1962-1967\end{array}$ & $\begin{array}{c}\text { Cohorte } \\
1975-1980\end{array}$ \\
\hline \multicolumn{5}{|l|}{ Profession du père } \\
\hline Ouvrier (réf.) & 0 & 0 & 0 & 0 \\
\hline Agriculteur & $-0,39$ & $1,07 * * *$ & $0,86^{* * *}$ & $0,74^{*}$ \\
\hline Artisan, commerçant, chef d'entreprise & 0,01 & $-0,14$ & $-0,11$ & 0,16 \\
\hline Cadre & 0,30 & 0,38 & $-0,20$ & 0,11 \\
\hline Profession intermédiaire & $-0,10$ & 0,10 & 0,02 & $-0,10$ \\
\hline Employé & $-0,76^{\star}$ & $-0,21$ & 0,18 & $-0,30$ \\
\hline Autre & 0,00 & 0,14 & $-0,31$ & 0,05 \\
\hline \multicolumn{5}{|l|}{ Diplôme du père } \\
\hline CAP, BEP, BEPC (réf.) & 0 & 0 & 0 & 0 \\
\hline Aucun diplôme ou CEP & 0,10 & $-0,11$ & $-0,16$ & 0,05 \\
\hline Baccalauréat & 0,13 & $0,58^{*}$ & 0,12 & $-0,02$ \\
\hline $\mathrm{Bac}+2$ & $0,66^{*}$ & $0,67 * * *$ & $-0,25$ & $-0,12$ \\
\hline Supérieur & $0,74 * * *$ & $1,12 * * *$ & $-0,39 *$ & $-0,35^{\star}$ \\
\hline \multicolumn{5}{|l|}{ Type de baccalauréat } \\
\hline Bac professionnel (réf.) & 0 & 0 & 0 & 0 \\
\hline Bac littéraire ou économique & 0,99 & $1,30 * * *$ & $-1,45^{\star \star *}$ & $-2,14 * \star \star$ \\
\hline Bac scientifique & $2,78^{* * *}$ & $2,58 * * *$ & $-0,70^{\star}$ & $-1,47 * * *$ \\
\hline Bac technologique & 0,03 & 0,57 & 0,13 & $-0,22$ \\
\hline \multicolumn{5}{|l|}{ Sexe } \\
\hline Homme (réf.) & 0 & 0 & 0 & 0 \\
\hline Femme & $-0,58^{* \star *}$ & $-0,46 * \star *$ & 0,15 & $-0,11$ \\
\hline Constante & $-3,70^{* * *}$ & $-4,19 * * *$ & $1,07 * * *$ & $1,64^{* * *}$ \\
\hline $\mathrm{G} 2$ & 1159 & 1289 & 1684 & 2104 \\
\hline Effectif & 2111 & 2454 & 1341 & 1733 \\
\hline \multicolumn{5}{|c|}{$\begin{array}{l}\text { Seuils de signification: }{ }^{*}: p \leq 0,05 ; * *: p \leq 0,01 ; * * *: p \leq 0,001 \text {. } \\
\text { Lecture: Le coefficient estimé pour les individus définis par la modalité active indique l'influence de cette } \\
\text { modalité, toutes choses étant égales par ailleurs, par rapport aux individus définis par la modalité de référence. } \\
\text { Le G2 (log-vraisemblance) permet d'estimer le pouvoir explicatif du modèle. } \\
\text { Source : calculs des auteurs d'après l'enquête FQP } 2003 \text {. }\end{array}$} \\
\hline
\end{tabular}

La première alternative dépend en premier lieu du type de baccalauréat possédé, les baccalauréats scientifiques dominant très largement tous les autres bacs. Mais à baccalauréat comparable, le niveau de diplôme du père (mais pas sa profession) exerce également une influence importante : l'orientation vers les filières d'« élite " est d'autant plus importante que le père est instruit. Les filles, quant à elles, s'orientent moins vers ces filières. Entre les deux cohortes, l'influence du niveau d'instruction du père a plutôt tendance à se renforcer et on voit apparaître un impact positif du fait d'être enfant d'agriculteur (ce qui 
est sans doute en rapport avec la modification sociologique de ce groupe pendant la période étudiée). La seconde alternative (pour la population n'ayant pas voulu ou pu accéder aux filières d'« élite ») n’apparaît pas liée à l'origine familiale, si ce n'est un léger impact négatif du niveau d'instruction paternelle et une tendance plus marquée des enfants d'agriculteurs à choisir une filière professionnelle plutôt qu'une université. C'est la série du baccalauréat qui entraîne la plus grosse différence : les bacheliers littéraires ou économiques sont de loin ceux qui s'orientent le moins vers les filières professionnelles, suivis des bacheliers scientifiques. Enfin, les filles, à baccalauréat comparable, ne se détournent pas particulièrement de ces filières.

En conclusion, on peut dire que l'accès aux différents types d'études reste marqué par des inégalités sociales et de genre, la première forme d'inégalité étant très liée au type de baccalauréat possédé pour ce qui concerne les filières professionnelles courtes. Il n'en va pas de même pour ce qui est de l'accès aux filières d'« élite »; en l'occurrence, l'origine sociale (en fait essentiellement le niveau d'instruction du père) joue un rôle spécifique, au-delà du baccalauréat possédé : les odds ratios relatifs à l'obtention d'un baccalauréat scientifique parmi les bacheliers généraux sont bien plus faibles que ceux qui concernent l'accès à une filière d'« élite » pour les bacheliers généraux. Concernant le sexe, le constat le plus significatif est la moindre tendance des filles à s'orienter, à baccalauréat égal, vers les filières d'« élite », et non leur réticence à poursuivre des études professionnalisées. Cette structure des " choix» évolue peu au cours de la période étudiée : alors que globalement (sur l'ensemble de la population), les chances d'accéder à l'enseignement supérieur (et à toutes ses filières) se sont égalisées, l'orientation des bacheliers tend plutôt à devenir plus inégalitaire, et en particulier, l'accès aux filières sélectives d'« élite » pour lesquelles on n'observe aucune tendance à la démocratisation avec des odds ratios qui restent, en fin de période, à des niveaux élevés (7), sans commune mesure avec ceux que l'on observe dans les autres filières.

\section{Le choix d'une spécialité, un choix horizontal ?}

Au niveau de l'enseignement supérieur, les choix sont finalisés : il serait réducteur de les considérer simplement comme inégaux, de par le prestige ou le niveau de salaire de la profession sur laquelle ils débouchent; ils sont aussi véritablement différents. On peut faire l'hypothèse que certains domaines d'études apparaissent plus attractifs à tel ou tel groupe social, parce que les enfants y seraient familiarisés à certaines valeurs, activités, un style de travail, etc. Certains chercheurs (par exemple, van de Werfhorst et al., 2003) font l'hypothèse que de manière générale, les inégalités, au niveau de l'enseignement supérieur, sont de plus en plus « horizontales » et que, par exemple, le droit ou la médecine attireraient plus spécifiquement les enfants de la classe supérieure, tandis que les métiers de l'enseignement ou de l'ingénierie seraient plus attractifs pour les enfants des classes populaires (tandis que certaines filières seraient plus « neutres», l'économie ou les sciences naturelles par exemple). 
Tableau 10. Évolution de l'accès des enfants d'ouvriers aux différentes spécialités d'études supérieures comparé à celui des enfants de cadres

\begin{tabular}{|c|c|c|c|c|c|}
\hline & \multicolumn{3}{|c|}{ Taux d'accès (en \%) } & \multicolumn{2}{|c|}{$\begin{array}{l}\text { Écart entre enfants } \\
\text { de cadres et enfants } \\
\text { d'ouvriers }\end{array}$} \\
\hline & Tous & $\begin{array}{l}\text { Enfants } \\
\text { de cadres }\end{array}$ & $\begin{array}{c}\text { Enfants } \\
\text { d'ouvriers }\end{array}$ & $\begin{array}{l}\text { Différences de } \\
\text { pourcentage } \\
\text { (en points) }\end{array}$ & $\begin{array}{l}\text { Odds } \\
\text { ratios }\end{array}$ \\
\hline \multicolumn{6}{|c|}{ Parmi les bacheliers } \\
\hline \multicolumn{6}{|l|}{ Cohorte 1962-1967 } \\
\hline Culture, arts, humanités & 19,7 & 17,0 & 21,9 & $-4,9$ & 0,7 \\
\hline Droit, économie, commerce & 31,8 & 28,4 & 33,8 & $-5,4$ & 0,8 \\
\hline Sciences & 16,3 & 23,1 & 9,9 & 13,2 & 2,7 \\
\hline Médecine, santé & 12,5 & 15,1 & 12,0 & 3,1 & 1,3 \\
\hline Ingénierie & 17,9 & 15,8 & 20,7 & $-4,9$ & 0,7 \\
\hline \multicolumn{6}{|l|}{ Cohorte 1975-1980 } \\
\hline Culture, arts, humanités & 22,0 & 20,8 & 20,8 & 0,0 & 1,0 \\
\hline Droit, économie, commerce & 32,6 & 28,6 & 36,6 & $-8,0$ & 0,7 \\
\hline Sciences & 22,2 & 28,4 & 18,5 & 9,9 & 1,7 \\
\hline Médecine, santé & 8,1 & 10,0 & 6,1 & 3,9 & 1,7 \\
\hline Ingénierie & 14,1 & 10,8 & 17,3 & $-6,5$ & 0,6 \\
\hline \multicolumn{6}{|c|}{ Parmi les bacheliers scientifiques } \\
\hline \multicolumn{6}{|l|}{ Cohorte 1962-1967 } \\
\hline Culture, arts, humanités & 5,7 & 5,5 & 5,0 & 0,5 & 1,1 \\
\hline Droit, économie, commerce & 14,9 & 13,8 & 8,4 & 5,4 & 1,7 \\
\hline Sciences & 34,7 & 37,6 & 30,5 & 7,1 & 1,4 \\
\hline Médecine, santé & 24,3 & 23,6 & 32,4 & $-8,8$ & 0,6 \\
\hline Ingénierie & 19,5 & 19,4 & 22,3 & $-2,9$ & 0,8 \\
\hline \multicolumn{6}{|l|}{ Cohorte $1975-1980$} \\
\hline Culture, arts, humanités & 5,8 & 7,7 & 5,5 & 2,2 & 1,4 \\
\hline Droit, économie, commerce & 11,3 & 11,7 & 9,4 & 2,3 & 1,3 \\
\hline Sciences & 50,5 & 49,7 & 50,2 & $-0,5$ & 1,0 \\
\hline Médecine, santé & 15,4 & 16,8 & 11,5 & 5,3 & 1,6 \\
\hline Ingénierie & 16,1 & 13,4 & 23,4 & $-10,0$ & 0,5 \\
\hline
\end{tabular}

Faut-il par conséquent, comme le fait volontiers la recherche européenne, faire l'hypothèse, à ce niveau, de choix avant tout " horizontaux », qui ne seraient pas hiérarchisés? On peut être sceptique, car il est difficile de faire la part, pour expliquer les choix, entre ce qui relèverait de préférences authentiques pour tel ou tel domaine d'étude ou pour la profession correspondante, d'un côté, et ce qui découlerait d'atouts inégaux dès le départ et d'un positionnement par rapport à des perspectives professionnelles inégales, de l'autre. Mais il n'en est pas moins intéressant de rechercher comment les étudiants se 
répartissent entre des domaines d'études qualitativement différents, et en ne tenant pas compte cette fois de la hiérarchie induite par les types d'études. Nous avons distingué 5 grands domaines : droit-économie-commerce, culturearts-humanités ${ }^{(19)}$, sciences, médecine et santé, ingénierie.

Dès lors que l'on ne tient plus compte du type d'études et donc de l'aspect « vertical » du choix, en regroupant par exemple au sein du domaine santé à la fois les études de médecine et toutes les formations paramédicales, ou encore, au sein de l'ingénierie, les écoles d'ingénieurs, les STS et les IUT, les choix des jeunes bacheliers apparaissent relativement peu marqués par leur origine sociale, et assez stables d'une cohorte à l'autre. Certes, les enfants de cadres sont davantage représentés dans le domaine des sciences et de la santé, alors que les enfants d'ouvriers sont particulièrement nombreux dans le champ de l'ingénierie et celui du droit-économie-commerce, le seul domaine où les deux groupes sont également présents étant la culture et les humanités. Mais les odds ratios sont relativement faibles: 1,7 pour les deux domaines où les enfants de cadres sont les plus présents, et 0,6 et 0,7 pour ceux où ils le sont le moins.

À ce niveau des spécialités choisies, on peut dégager deux évolutions notables entre les cohortes. Dans le champ médical, les filières courtes professionnelles comme les filières longues ont tendance à devenir plus sélectives socialement, ce qui s'explique aisément dans le second cas compte tenu du caractère de plus en plus sélectif des études médicales, avec l'instauration d'un numerus clausus en 1971. En sciences, on observe au contraire une tendance à la démocratisation, sachant que cette filière reste la plus sélective socialement avec la médecine. Les autres tendances sont très ténues : le droit-économie et l'ingénierie tendent à devenir un peu plus populaires (peut-être parce que les baccalauréats technologiques et professionnels mènent préférentiellement vers ces domaines), alors que la culture et les humanités le deviennent un peu moins, avec un odds ratio de 1 en fin de période.

Ces tendances ne sont pas démenties si on isole les bacheliers scientifiques, mais les orientations de ces jeunes qui sont les plus sélectionnés scolairement restent marquées par de fortes différenciations sociales $\left(2^{\mathrm{e}}\right.$ partie du tableau 10$)$. Une opposition forte apparaît entre le secteur médical dont le caractère socialement sélectif se renforce sur la période (de même que les orientations vers la culture et les humanités, certes moins nombreuses) d'une part, et les orientations vers l'ingénierie qui sont les plus populaires et le sont encore un peu plus au fil du temps d'autre part.

Pour mieux cerner ces tendances, nous avons estimé des modèles multinomiaux pour quatre grands domaines de choix (tableau 11). Seuls les modèles estimés sur la cohorte 2 (1975-1980) sont présentés ici, mais le commentaire soulignera l'évolution avec la première cohorte. 
Tableau 11. Analyse de l'accès aux différentes filières de l'enseignement supérieur, cohorte 1975-1980 (coefficients des modèles multinomiaux)

\begin{tabular}{|c|c|c|c|c|c|c|}
\hline & \multicolumn{3}{|c|}{$\begin{array}{l}\text { Droit, } \\
\text { économie vs humanités, } \\
\text { culture }\end{array}$} & \multicolumn{3}{|c|}{$\begin{array}{l}\text { Sciences vs humanités, } \\
\text { culture }\end{array}$} \\
\hline & $\begin{array}{c}\text { Modèle } \\
1\end{array}$ & $\begin{array}{c}\text { Modèle } \\
2\end{array}$ & $\begin{array}{l}\text { Modèle } \\
3\end{array}$ & $\begin{array}{c}\text { Modèle } \\
1\end{array}$ & $\begin{array}{l}\text { Modèle } \\
2\end{array}$ & $\begin{array}{l}\text { Modèle } \\
3\end{array}$ \\
\hline \multicolumn{7}{|l|}{ Origine sociale } \\
\hline Non-cadre (réf.) & 0 & 0 & 0 & 0 & 0 & 0 \\
\hline Cadre & 0,18 & $0,46^{* *}$ & $0,46^{* *}$ & 0,36 & 0,35 & 0,30 \\
\hline \multicolumn{7}{|l|}{ Sexe } \\
\hline Homme (réf.) & 0 & 0 & 0 & 0 & 0 & 0 \\
\hline Femme & $-0,69 * * *$ & $0,63 * * *$ & $-0,63 * * *$ & $-1,58 * * *$ & $-1,59 * * *$ & $-1,60 * * *$ \\
\hline \multicolumn{7}{|l|}{ Type de baccalauréat } \\
\hline Baccalauréat général (réf.) & & 0 & 0 & & 0 & 0 \\
\hline Baccalauréat professionnel & & $1,63 * * *$ & $1,62 * * *$ & & $-0,13$ & $-0,23$ \\
\hline Baccalauréat technologique & & $1,46 * * *$ & $1,46 * * *$ & & $-0,19$ & $-0,18$ \\
\hline \multicolumn{7}{|l|}{ Mention au baccalauréat } \\
\hline Pas de mention (réf.) & & & 0 & & & 0 \\
\hline Mention & & & 0,08 & & & $0,48^{* * *}$ \\
\hline \multirow[t]{3}{*}{ Constante } & $0,99 * * *$ & $0,41 * *$ & $0,39 *$ & $0,95 * * *$ & $0,98 * * *$ & $0,83 * * *$ \\
\hline & \multicolumn{3}{|c|}{$\begin{array}{l}\text { Médecine, } \\
\text { santé vs humanités, culture }\end{array}$} & \multicolumn{3}{|c|}{$\begin{array}{l}\text { Ingénierie vs humanités, } \\
\text { culture }\end{array}$} \\
\hline & $\begin{array}{c}\text { Modèle } \\
1\end{array}$ & $\begin{array}{c}\text { Modèle } \\
2\end{array}$ & $\begin{array}{l}\text { Modèle } \\
3\end{array}$ & $\begin{array}{c}\text { Modèle } \\
1\end{array}$ & $\begin{array}{l}\text { Modèle } \\
2\end{array}$ & $\begin{array}{l}\text { Modèle } \\
3\end{array}$ \\
\hline \multicolumn{7}{|l|}{ Origine sociale } \\
\hline Non-cadre (réf.) & 0 & 0 & 0 & 0 & 0 & 0 \\
\hline Cadre & $0,57^{* *}$ & $0,64^{* *}$ & $0,64^{* *}$ & $-0,30$ & 0,11 & 0,08 \\
\hline \multicolumn{7}{|l|}{ Sexe } \\
\hline Homme (réf.) & 0 & 0 & 0 & 0 & 0 & 0 \\
\hline Femme & 0,23 & 0,25 & 0,25 & $-3,07 * * *$ & $-3,00 * * *$ & $-3,00 * * *$ \\
\hline \multicolumn{7}{|l|}{ Type de baccalauréat } \\
\hline Baccalauréat général (réf.) & & 0 & 0 & & 0 & 0 \\
\hline Baccalauréat professionnel & & 0,36 & 0,36 & & $1,82 * * *$ & $1,74^{* * *}$ \\
\hline Baccalauréat technologique & & 0,56 & 0,56 & & $2,10 * * *$ & $2,12 * * *$ \\
\hline \multicolumn{7}{|l|}{ Mention au baccalauréat } \\
\hline Pas de mention (réf.) & & & 0 & & & 0 \\
\hline Mention & & & $-0,01$ & & & 0,39 \\
\hline Constante & $-1,26^{* * *}$ & $-1,45^{* * *}$ & $-1,44^{* * *}$ & $1,24 * * *$ & 0,33 & 0,21 \\
\hline \multicolumn{7}{|l|}{$\begin{array}{l}R^{2}=0,152 \\
\text { Effectif = } 1123\end{array}$} \\
\hline \multicolumn{7}{|c|}{$\begin{array}{l}\text { Seuils de signification : * }: p \leq 0,05 ;{ }^{* *}: p \leq 0,01 ;{ }^{* *}: p \leq 0,001 \text {. } \\
\text { Lecture : les modèles } 1 \text { sont estimés sur les variables de base (sexe et origine sociale). Les modèles } 2 \text { ajoutent } \\
\text { la variable type de bac, puis les modèles } 3 \text { la mention. } \\
\text { Source : calculs des auteurs d'après l'enquête FQP } 2003 \text {. }\end{array}$} \\
\hline
\end{tabular}


En ce qui concerne le choix du droit, de l'économie ou du commerce (tous types d'études réunis), plutôt que des humanités ou de la culture, c'est un choix fait plus fréquemment, dans la première cohorte, par les garçons, par les titulaires d'un baccalauréat technologique, et moins souvent par les bacheliers ayant obtenu une mention. Ces facteurs étant contrôlés, il n'y a pas d'effet de l'origine sociale. Une évolution s'observe dans la seconde cohorte. Linfluence du baccalauréat possédé se fait plus forte, les bacheliers technologiques et professionnels choisissant plus souvent ce champ (sans doute plus le commerce que les disciplines universitaires du droit et des sciences économiques). On note aussi que les filles sont encore moins portées à choisir ce domaine, et que par ailleurs l'obtention d'une mention ne marque plus (négativement) ce choix, qui serait donc moins souvent dédaigné par les jeunes bacheliers dotés d'un bon niveau académique.

Pour ce qui est du choix des sciences, il est fait préférentiellement, en début de période, par les garçons qui possèdent un baccalauréat général (mais pas plus souvent une mention), et il est marqué à ce stade par des inégalités sociales. Là encore, des changements se sont produits entre les deux cohortes : les élèves de bon niveau académique se montrent plus attirés par cette orientation (le rôle de la mention devient significatif), mais l'influence jusqu'alors négative de la possession d'un baccalauréat technologique ou professionnel décroît ; par ailleurs, l'influence de l'origine sociale cesse d'être significative ; le domaine scientifique semble devenir un choix de bons élèves de milieu populaire. En revanche, les filles restent toujours aussi réticentes à choisir ce domaine.

Concernant le domaine médical (filières courtes et longues réunies), on observe que dans la première cohorte, aucune des caractéristiques prises en compte (sexe, origine sociale, bac, mention...) n'exerce d'influence significative. Dans la seconde cohorte, une origine sociale élevée devient un facteur significatif, ce qui est cohérent avec l'accroissement de la sélectivité sociale de cette filière.

Enfin, en ce qui concerne l'ingénierie (là encore en mélangeant les filières courtes, de type STS-IUT, et longues, de type grandes écoles), ce domaine apparaît principalement comme un choix de garçons dotés d'un baccalauréat technologique; ni la mention ni l'origine sociale n'ont d'influence significative. Et cela ne change pas entre les deux cohortes ; tout au plus note-t-on un impact plus marqué des baccalauréats technologiques et professionnels et le fait que les filles sont encore moins enclines à choisir ce domaine.

De manière globale, si on prend en compte l'ensemble des choix (ce que permettent les modèles multinomiaux), le type de baccalauréat possédé apparaît important surtout dans deux domaines, droit-économie-commerce et ingénierie. Lorigine sociale joue fortement en médecine ainsi qu'en droit-économie-commerce ; la mention obtenue ne joue qu'en sciences. Enfin, le fait d'être une femme (plutôt qu'un homme) est associé négativement et de manière significative au choix de l'ingénierie, des sciences et à un degré moindre à celui du droit-économiecommerce alors qu'il est associé positivement au domaine humanités-culture (il n'y a pas de corrélation significative avec le choix des carrières de la santé). 
Au total, certains de ces résultats ne sont pas contradictoires avec les hypothèses de la sociologie européenne, notamment le fait que c'est dans le domaine de l'ingénierie et des sciences que l'influence du milieu social est la plus faible, ou, pour dire les choses autrement, que les enfants d'ouvriers se distinguent le moins des enfants de cadres. Faut-il aller jusqu'à dire que ceci traduirait un " goût populaire » marqué par l'expérience du travail industriel des parents (comme le suggère van de Werfhorst), alors que le choix du domaine de la santé résulterait d'une proximité d'expérience avec celle de parents cadres ? Nos résultats ne permettent pas de soutenir la thèse selon laquelle il y aurait des goûts foncièrement différents selon les milieux sociaux : les odds ratios attachés aux différents domaines d'études sont en effet relativement faibles. De plus, cette perspective donnant priorité à la notion de choix « horizontal » où le goût prime sur le classement n'explique pas certaines contradictions : comment comprendre que les filières d'« élite» deviennent socialement plus sélectives alors que le domaine de l'ingénierie le serait moins? Il nous semble plus justifié, dans le contexte français en tout cas, de prendre en compte à la fois la hiérarchie des filières et les domaines: si les préférences (et, pourquoi pas, des préférences différentes selon les groupes sociaux) peuvent orienter vers tel ou tel domaine, les atouts scolaires et socio-économiques des uns et des autres détermineraient dans quel type de filière ces préférences vont pouvoir se concrétiser (école d'ingénieur ou BTS, médecine ou formation paramédicale, etc.).

Il est clair que si les préférences socialement héritées étaient le facteur essentiel, on devrait observer ces attirances quel que soit le type d'études ; on aurait donc une surreprésentation des enfants d'ouvriers dans tous les types de filières menant vers l'ingénierie, ou encore une surreprésentation des enfants de cadres dans toutes celles du domaine de la santé. D’ores et déjà, on peut souligner que les écarts mesurés par les odds ratios sont plus importants en ce qui concerne le type de filière qu'entre les différentes disciplines. Il semble donc bien que le classement dans une hiérarchie de filières l'emporte sur des préférences purement horizontales (tableau 12).

En effet, les odds ratios les plus élevés concernent systématiquement les études sélectives ou de troisième cycle universitaire dans tous les domaines et, réciproquement, les études les plus ouvertes socialement sont les filières professionnelles courtes, là encore dans tous les domaines. Il se confirme que le secteur de la santé est devenu plus sélectif, notamment pour la filière longue, mais aussi à un degré moindre pour les autres filières, alors qu'au contraire, la sélection sociale a diminué en sciences dans toutes les filières. On remarque dans la seconde cohorte la percée des enfants d'ouvriers dans les filières universitaires du domaine de l'ingénierie, pratiquement inexistantes en début de période et qui se sont fortement développées (avec notamment les maîtrises de sciences et techniques). En fin de période, ce domaine est très nettement le plus ouvert socialement par rapport aux autres domaines. 
Tableau 12. Origine sociale des diplômés du supérieur selon la discipline et le type de filière

\begin{tabular}{|c|c|c|c|c|}
\hline & \multicolumn{2}{|c|}{ Part (en \%) } & \multicolumn{2}{|c|}{$\begin{array}{l}\text { Écart entre enfants de } \\
\text { cadres et d'ouvriers }\end{array}$} \\
\hline & $\begin{array}{l}\text { Enfants de } \\
\text { cadres }\end{array}$ & $\begin{array}{l}\text { Enfants } \\
\text { d'ouvriers }\end{array}$ & $\begin{array}{c}\text { Différences de } \\
\text { pourcentage } \\
\text { (en points) }\end{array}$ & Odds ratios \\
\hline \multicolumn{5}{|c|}{ Cohorte 1962-1967 } \\
\hline \multicolumn{5}{|l|}{ Culture, humanités } \\
\hline Université & 21,3 & 22,9 & $-1,6$ & 0,9 \\
\hline Filières d'élite & 30,2 & 8,1 & 22,1 & 4,9 \\
\hline \multicolumn{5}{|l|}{ Droit, économie, commerce } \\
\hline STS, IUT & 16,8 & 23,9 & $-7,1$ & 0,6 \\
\hline Université & 23,7 & 21,3 & 2,4 & 1,1 \\
\hline Filières d'élite et filières longues & 40,1 & 4,4 & 35,7 & 14,5 \\
\hline \multicolumn{5}{|l|}{ Sciences } \\
\hline STS, IUT & 22,9 & 17,6 & 5,3 & 1,4 \\
\hline Université & 32,6 & 10,7 & 21,9 & 4,0 \\
\hline Filières d'élite et filières longues & 44,0 & 8,4 & 35,6 & 8,6 \\
\hline \multicolumn{5}{|l|}{ Santé } \\
\hline STS, IUT & 18,6 & 23,2 & $-4,6$ & 0,8 \\
\hline Université & 40,4 & 16,0 & 24,4 & 3,6 \\
\hline Filières d'élite et filières longues & 41,8 & 15,0 & 26,8 & 4,1 \\
\hline \multicolumn{5}{|l|}{ Ingénierie } \\
\hline STS, IUT & 15,4 & 24,3 & $-8,9$ & 0,6 \\
\hline Filières d'élite et filières longues & 46,8 & 15,1 & 31,7 & 4,9 \\
\hline \multicolumn{5}{|c|}{ Cohorte 1975-1980 } \\
\hline \multicolumn{5}{|l|}{ Culture, humanités } \\
\hline Université & 20,7 & 20,3 & 0,4 & 1,0 \\
\hline Filières d'élite & 36,1 & 6,5 & 29,6 & 8,1 \\
\hline \multicolumn{5}{|l|}{ Droit, économie, commerce } \\
\hline STS, IUT & 17,6 & 30,7 & $-13,1$ & 0,5 \\
\hline Université & 29,4 & 19,2 & 10,2 & 1,8 \\
\hline Filières d'élite et filières longues & 41,0 & 8,9 & 32,1 & 7,1 \\
\hline \multicolumn{5}{|l|}{ Sciences } \\
\hline STS, IUT & 19,5 & 24,4 & $-4,9$ & 0,8 \\
\hline Université & 28,1 & 16,7 & 11,4 & 1,9 \\
\hline Filières d'élite et filières longues & 50,7 & 13,1 & 37,6 & 6,8 \\
\hline \multicolumn{5}{|l|}{ Santé } \\
\hline STS, IUT & 21,4 & 17,0 & 4,4 & 1,3 \\
\hline Université & 43,9 & 12,8 & 31,1 & 5,3 \\
\hline Filières d'élite et filières longues & 62,0 & 11,5 & 50,5 & 12,6 \\
\hline \multicolumn{5}{|l|}{ Ingénierie } \\
\hline STS, IUT & 13,6 & 30,6 & $-17,0$ & 0,4 \\
\hline Université & 21,9 & 43,9 & $-22,0$ & 0,4 \\
\hline Filières d'élite et filières longues & 30,5 & 9,4 & 21,1 & 4,2 \\
\hline
\end{tabular}


Derrière ces évolutions et la structure des inégalités constatées - les filières étant socialement plus sélectives que les spécialités -, il y a évidemment des stratégies dont on peut penser qu'elles intègrent des perspectives d'insertion professionnelle. Sans engager ici une analyse précise du « rendement » des différentes filières, notons simplement qu'au vu de nos analyses des données de l'enquête FQP, les chances d'obtenir un premier emploi de cadre sont déterminées avant tout par le type d'études et secondairement par leur spécialité. Les filières d'élite surpassent à cet égard très nettement les filières universitaires et bien sûr les filières professionnelles courtes. On comprend donc aisément que la compétition sociale porte avant tout sur l'accès aux grandes écoles et aux classes qui y préparent.

\section{Conclusion}

Cette analyse de l'évolution des inégalités face à l'enseignement supérieur dans la période de très fort développement de l'enseignement secondaire qu'a constitué la décennie 1985-1995 confirme que l'ouverture quantitative n'est pas une condition suffisante à l'atténuation des inégalités. L'indéniable démocratisation du baccalauréat (démocratisation égalisatrice, pour reprendre l'expression de Merle) se traduit par une démocratisation « uniforme » de l'accès à l'enseignement supérieur, qui laisse les écarts inchangés. S'ajoute à cela la démocratisation ségrégative qui a marqué l'accès au bac, avec le recrutement de «nouveaux bacheliers » de milieu populaire au sortir d'un brevet d'études professionnelles, pour les conduire vers les baccalauréats professionnels. Compte tenu de la force des articulations entre enseignement secondaire et supérieur en France, ce phénomène agit comme une limitation quant à la « rentabilisation possible » du baccalauréat dans l'enseignement supérieur. Il faut compter aussi avec les évolutions de l'offre et avec une stagnation de la part relative des filières dites d'« élite » (malthusianisme compréhensible dès lors qu'elles veulent préserver leurs débouchés), qui rend la compétition de plus en plus dure. Au-delà de ces phénomènes qui vont contraindre les flux, il faut compter enfin avec les stratégies des jeunes, notamment ceux qui ont le plus d'atouts en main et qui cherchent à rester en tête.

On observe à cet égard, pour l'enseignement supérieur, un schéma assez classique ; une digue qui cède à un niveau, une rétention qui se fait un peu plus loin, avant que cette nouvelle digue cède à son tour : c'est ainsi que l'ouverture de la $6^{\text {e }}$ à tous fut suivie d'une plus forte sélectivité des carrières scolaires au collège, que la disparition ultérieure du palier d'orientation en fin de $5^{\mathrm{e}}$ a été suivie d'une orientation plus sélective en fin de $3^{\mathrm{e}}$ puis en fin de $2^{\text {nde }}$. Aujourd'hui, cette «vague " parvient aux portes de l'université. Mais à ce niveau, les jeunes sont censés viser un diplôme terminal, leur permettant de s'insérer. La vague ne peut aller sans cesse plus loin, les inégalités étant simplement repoussées pour un temps, car il faudra bien que se fasse une articulation avec les emplois, dont l'évolution n'a aucune raison d'être en phase avec celle des flux de diplômés. 
On voit bien les limites d'une politique éducative (et/ou des jeux stratégiques) qui ne fait que déplacer les inégalités de formation, sans avoir évidemment prise sur les emplois qui suivront. Il s'ensuit une dévalorisation relative des titres, et un effet de ciseau bien mis en évidence par Forsé (1997 ; voir aussi Chauvel, 1998, et Duru-Bellat, 2006) : les enfants d'ouvriers deviennent plus souvent bacheliers, mais vu le type de baccalauréat auquel ils accèdent et les études supérieures auxquelles ce baccalauréat permet d'accéder, ils arrivent sur le marché du travail avec des diplômes qui leur laissent une probabilité élevée d'être ouvriers ou employés. On comprend, à l'autre bout du spectre, cette polarisation des enfants de cadres sur les filières d'« élite», qui parviennent à préserver leur valeur sur le marché du travail et à garantir l'accès à un emploi de cadre. Au-delà des analyses sur les préférences des enfants de telle ou telle origine sociale et la montée de différenciations horizontales, il y a bien une compétition pour des places inégales, qui se joue très tôt puisque dès la fin de la $3^{\mathrm{e}}$, les élèves les plus en difficulté (qui le sont déjà souvent depuis le primaire) s'engagent dans un cycle court technologique qui les aiguillera vers un baccalauréat professionnel, alors que d'autres entreront en $2^{\text {nde }}$ avec les options laissant espérer un baccalauréat général.

Promouvoir l'égalité des chances ne peut se limiter à jouer à la marge sur les processus de sélection dans les filières d'« élite » par différents systèmes de discrimination positive (comme les procédures d'admission spéciale pour les élèves des lycées défavorisés) alors que, par définition, celles-ci resteront toujours quantitativement restreintes. Il conviendrait de jouer bien en amont, sur les inégalités de réussite précoce et donc sur les chances d'accéder aux voies scolaires les mieux adaptées à ces filières (en l'occurrence l'accès aux baccalauréats généraux). De multiples actions en aval sont bien sûr concevables, telles que favoriser les formations tout au long de la vie qui sont, en France, particulièrement rares, ce qui donne encore plus de portée aux inégalités scellées très tôt dans l'éducation initiale.

\section{(1)}

\section{RÉFÉRENCES}

Albouy V., WANECQ T., 2003, « Les inégalités sociales d'accès aux grandes écoles », Économie et Statistique, $\mathrm{n}^{\circ} 361$, p. 27-52

ARUM R., GAMORAN A., SHAVIT Y., 2007, « Inclusion and diversion in higher education : A study of expansion and stratification in 15 countries » in Shavit Y., Arum R., Gamoran A. (éd.), Stratification in Higher Education: A Comparative Study, Stanford (CA), Stanford University Press, p. 1-35.

BRINBAUM Y., KIEFFER A., 2005, « D’une génération à l'autre, les aspirations éducatives des familles immigrées : ambition et persévérance », Éducation et Formations, $\mathrm{n}^{\circ} 72$, p. $53-75$. 
CHAuvel L., 1998, « La seconde explosion scolaire : diffusion des diplômes, structure sociale et valeur des titres », Revue de l'OFCE, $\mathrm{n}^{\circ} 66$, p. 5-36.

CHERKAOUI M., 1982, Les changements du système éducatif en France : 1950-1980, Paris, PUF.

CONVERT B., 2006, Les impasses de la démocratisation scolaire, Paris, Éditions Raisons d'Agir, 93 p.

CONVERT B., PInET M., 1989, « Les classes terminales et leur public », Revue française de sociologie, 30(2), p. 211-234.

Duru-Bellat M., 2002, Les inégalités sociales à l'école. Genèse et mythes, Paris, PUF, $256 \mathrm{p}$.

Duru-Bellat M., 2006, L’inflation scolaire. Les désillusions de la méritocratie, Paris, Éditions du Seuil.

Duru-Bellat M., KiefFer A., 2000, « La démocratisation de l'enseignement en France : polémiques autour d'une question d'actualité », Population, 55(1), p. 51-80.

Duru-Bellat M., KiefFer A., MARry C., 2001, « La dynamique des scolarités des filles : le double handicap questionné ", Revue française de sociologie, 42(2), p. 251-280.

FORSÉ M., 1997, «La diminution de l'inégalité des chances scolaires ne suffit pas à réduire l'inégalité des chances sociales », Revue de L'OFCE, n 63, p. 229-239.

GOUX D., MAURIN É., 1997, « Démocratisation de l'école et persistance des inégalités », Économie et Statistique, $\mathrm{n}^{\circ}$ 306, p. 27-33.

MANZO G., 2006, Analyse comparée de la stratification éducative en France et en Italie dans les années quatre-vingt-dix et au cours $d u X X^{e}$ siècle. De la description statistique à l'explication par simulation "individus-centrée ", Thèse de doctorat en sciences sociales, Université de Paris IV-Sorbonne.

MARE R. D., 1981, "Change and stability in educational stratification », American Sociological Review, 46, p. 72-87.

MERLE P., 1996, «Les transformations sociodémographiques des filières de l'enseignement supérieur de 1985 à 1995. Essai d'interprétation », Population, 51(6), p. 1181-1210.

MERLE P., 2000, « Le concept de démocratisation de l'institution scolaire : une typologie et sa mise à l'épreuve », Population, 55(1), p. 15-50.

MERLE P., 2002, « Démocratisation ou accroissement des inégalités scolaires ? L'exemple de l'évolution de la durée des études en France (1988-1998) » Population-F, 57(4-5), p. 633-660.

SElZ M., VAlLET L.-A., 2006, « La démocratisation de l'enseignement et son paradoxe apparent », in Données sociales, La société française, Insee, p. 101-107.

VALLET L.-A., 2001, « La mesure des évolutions des inégalités sociales et scolaires en longue période », in Boudon R., Bulle N., Cherkaoui M. (dir.), École et société : les paradoxes de la démocratie, Paris, PUF, p. 187-206.

VAn De Werfhorst H., Sullivan A., Cheung S. Y., 2003, « Social class, ability choice of subject in secondary education in Britain », British Educational Research Journal, 29 (1), p. 41-62. 


\section{Marie DuRU-Bellat, ANNICK KiefFer • Du baccalauréAt À l'Enseignement SUPÉRIEUR}

\section{EN FRANCE : DÉPLACEMENT ET RECOMPOSITION DES INÉGALITÉS}

La dernière enquête FQP réalisée par I'Insee en 2003 permet d'évaluer les transformations intervenues au niveau de l'enseignement supérieur dans deux groupes de cohortes encadrant la période d'expansion très forte de l'accès au baccalauréat entre 1985 et 1995. Comment cette ouverture a-t-elle affecté les inégalités sociales d'accès et de réussite dans l'enseignement supérieur ? L'indéniable démocratisation du baccalauréat se traduit par une démocratisation plus limitée de l'accès au supérieur. En effet, la première vague de démocratisation s'est traduite par une concentration accrue des nouveaux bacheliers de milieu populaire dans les baccalauréats professionnels ; compte tenu de la force des articulations entre enseignement secondaire et supérieur en France, ce phénomène a induit une limitation des possibilités d'orientation dans l'enseignement supérieur. En particulier, l'accès aux filières d'élite n'a connu aucune démocratisation parmi les bacheliers, alors que les filières professionnelles courtes et les filières universitaires s'ouvraient. Ceci limite la portée de la démocratisation de l'enseignement à ce niveau en termes de mobilité sociale, puisque de plus en plus, les débouchés ne dépendent pas du niveau de diplôme, mais du type de filière suivie.

\section{Marie Duru-Bellat, Annick Kieffer • From the Baccalauréat to Higher Education IN FRANCE: SHIFTING INEQUALITIES}

INSEE's most recent survey in 2003 makes it possible to evaluate changes in higher education in two birth cohort groups at either end of the period of rapid expansion in access to the baccalauréat (upper secondary exit examination) from 1985 to 1995 . We examine how this opening up affected social inequalities in access to and success in higher education. The undeniable democratization of the baccalauréat has been followed by a more limited democratization in access to higher education. The first wave concentrated new working-class students in vocational baccalauréats; but owing to the strong links between secondary and higher education in France, these students' options in higher education have been restricted as a consequence. In particular, access to the selective elite grandes écoles has seen no democratization among holders of the baccalauréat, whereas shorter vocational courses and non-selective university courses have opened up. This limits the effect on social mobility of democratization of education at this level, because career opportunities increasingly depend not so much on the level of qualification but on the subjects chosen.

\section{Marie DuRu-Bellat, ANNiCK KiefFer • Del bachillerato a la enseñanza superior en FRANCIA : DESPLAZAMIENTO Y RECOMPOSICIÓN DE DESIGUALDADES}

La última encuesta FQP realizada por el Insee en 2003 permite evaluar las transformaciones que han tenido lugar a nivel de la enseñanza superior en dos grupos de cohortes que enmarcan el período de expansión muy fuerte del acceso al bachillerato (baccalauréat) entre 1985 y 1995. La pregunta que se plantea es la de saber cómo esta apertura ha influido en las desigualdades sociales de acceso y de éxito en la enseñanza superior. La innegable democratización del bachillerato se traduce en una democratización más limitada del acceso a los estudios superiores. En efecto, la primera ola de democratización se reflejó en una concentración incrementada de los nuevos bachilleres de medio popular en los bachilleratos profesionales; teniendo en cuenta la fuerza de las articulaciones entre enseñanza secundaria y superior en Francia, este fenómeno ha inducido una limitación de las posibilidades de orientación en la enseñanza superior. En particular, el acceso a las carreras más selectas no ha experimentado democratización alguna entre los bachilleres, mientras que las carreras profesionales cortas y la carreras universitarias se abrían. Ello limita el alcance de la democratización de la enseñanza a este nivel en términos de movilidad social, puesto que cada vez más, las salidas y oportunidades no dependen del nivel de diploma sino del tipo de itinerario seguida. 